Document downloaded from:

http://hdl.handle.net/10251/167217

This paper must be cited as:

Alvarez, JO.; Penaranda-Foix, FL.; Catalá Civera, JM.; Gutiérrez Cano, JD. (2020). Permittivity Spectrum of Low-Loss Liquid and Powder Geomaterials Using Multipoint Reentrant Cavities. IEEE Transactions on Geoscience and Remote Sensing. 58(5):30973112. https://doi.org/10.1109/TGRS.2019.2948052

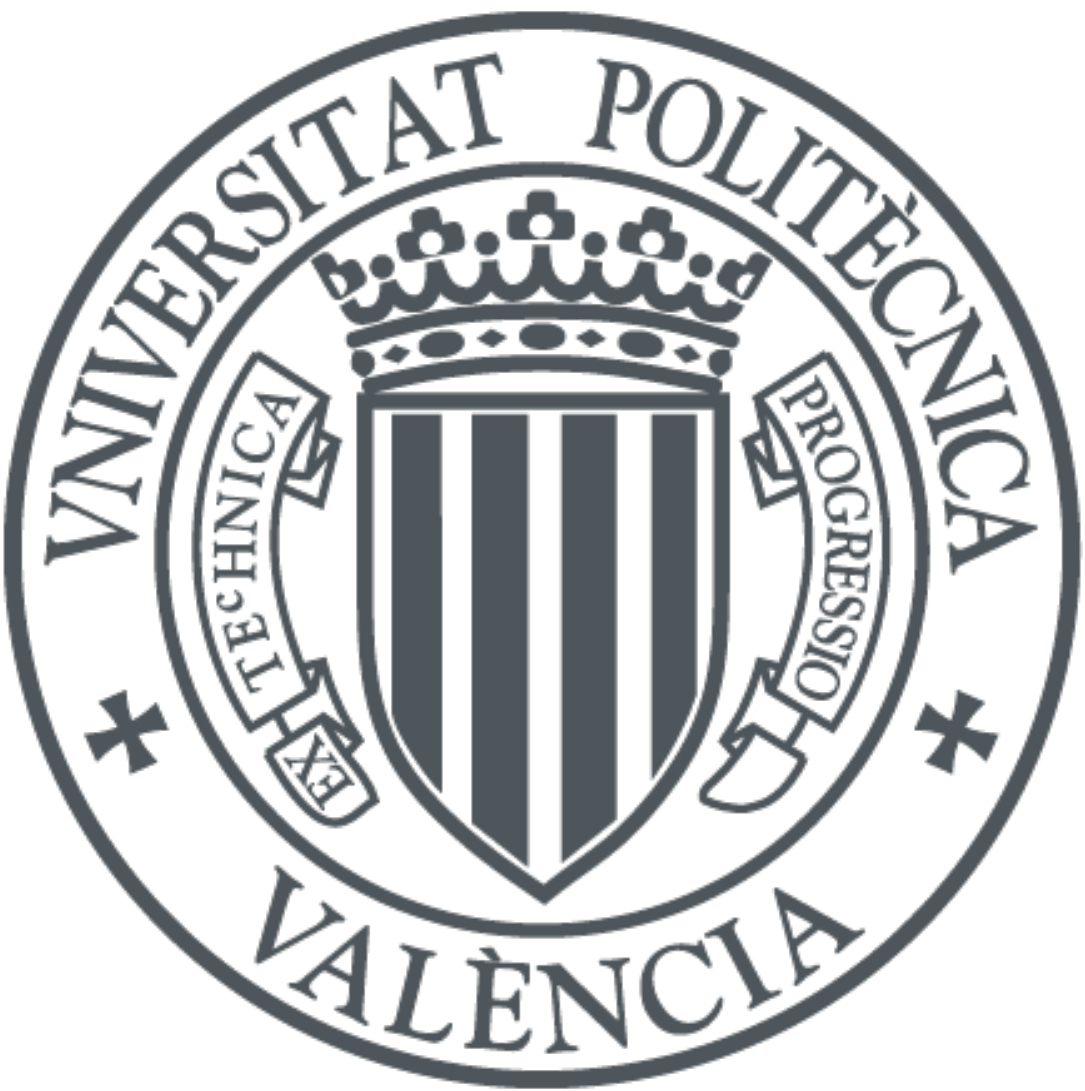

The final publication is available at

https://doi.org/10.1109/TGRS.2019.2948052

Copyright Institute of Electrical and Electronics Engineers

Additional Information 


\title{
Permittivity Spectrum of Low-Loss Liquid and Powder Geomaterials Using Multipoint Re-entrant Cavities
}

\author{
Jose Oliverio Alvarez, Senior Member, IEEE, Felipe L. Peñaranda-Foix, Senior Member, IEEE, Jose \\ M. Catala-Civera, Senior Member, IEEE, and Jose D. Gutierrez-Cano
}

\begin{abstract}
-
Permittivity is a useful tool to characterize the composition and quality of many geomaterials. In general, non-resonant permittivity measurement methods exhibit a higher degree of uncertainty than their resonant counterparts. In resonant measurements, the reduction in uncertainty comes typically with a loss in broadband. This work describes the theory, design, and application of multipoint coaxial re-entrant resonant cavities applied to low-loss geomaterials at different temperatures. Specifically, a full wave method based on circuit analysis is developed and applied for a circular corrugated waveguide. Moreover, the mode-matching method is applied to calculate the Generalized Admittance Matrix (GAM). Two multipoint cavities and software were built and validated. The first cavity has five resonant frequencies, between $170 \mathrm{MHz}$ to $2.3 \mathrm{GHz}$, and the second has four resonant frequencies, between $1.3 \mathrm{GHz}$ and 8.6 GHz. Thus, this method allows for "broadband-resonant" measurements. Permittivity values of liquid hydrocarbons, powdered kerogen, and pyrite are shown.
\end{abstract}

Index Terms - Permittivity, microwave measurements, coaxial cavity, crude oil, oil spill, kerogen, pyrite, dielectric loaded waveguides, circular corrugated waveguides.

\section{INTRODUCTION}

$\mathrm{T}$ he quality, geothermal maturity, and chemical composition of low-loss geomaterials, such as reservoir hydrocarbons or kerogen, are determined by laboratory analyses. Results from these analyses may take days or weeks, giving little to no room to take proactive measures if a problem, such as reduction in crude oil quality, is detected.

Microwave measurements provide values for the relative complex permittivity of a material under test (MUT). The complex permittivity is used to characterize important properties of fluids [1-6] such as density and chemical composition. The relative complex permittivity is given by [7]:

$$
\varepsilon(f, T, C)=\varepsilon^{\prime}(f, T, C)-j \varepsilon^{\prime \prime}(f, T, C),
$$

where $f$ is the frequency, $T$ is the temperature and $C$ is the chemical composition. The imaginary part is given by:

J. O. Alvarez is with the Sensors Development Team, Aramco Services Company, Aramco Research Center - Houston, Houston, TX 77084 USA (email: oliverio.alvarez@aramcoservices.com).

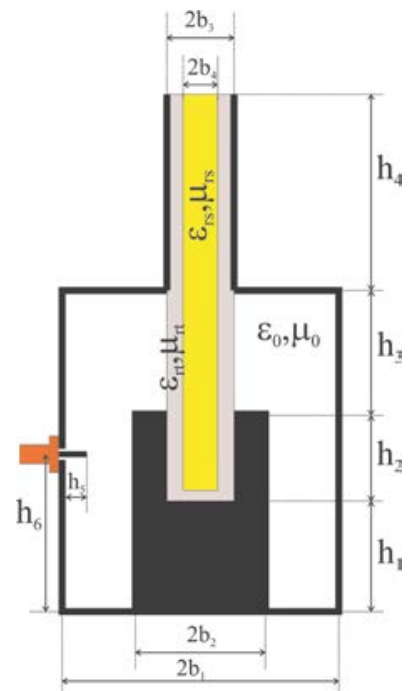

Fig. 1: Geometry of the re-entrant cavity with vials. Orange represents the coaxial excitation, gray is the tube that contains sample, yellow is the sample to be measured, black is metals and white is air.

$$
\varepsilon^{\prime \prime}(f, T, C)=\varepsilon_{d}^{\prime \prime}(f, T, C)+\sigma_{D C}(T, C) / 2 \pi f \varepsilon_{0} .
$$

The first term represents the dielectric losses and the second term the conductive losses. $\sigma_{\mathrm{DC}}$ is the DC conductivity and $\varepsilon_{0}$ is the permittivity of vacuum.

Depending on their nature, permittivity measurements can be broadband or resonant. Broadband measurements, given their reflection/transmission nature, have higher uncertainty and low accuracy for low-loss materials. As opposed to resonant methods, which provide high accuracy and lower uncertainty for the same materials, but at single frequency.

In this work we present a system of multipoint, re-entrant microwave cavities. These cavities are capable of measuring a discrete number of permittivity values over a large frequency range using harmonic resonant modes that are not mixed with other higher order modes. Moreover, the present study will show that two or more cavities can be designed for different

F. L. Peñaranda-Foix, J. M. Catala-Civera and J. D. Gutierrez-Cano are with the Instituto de Tecnologías de la Información y las Comunicaciones (ITACA), Universitat Politecnica de Valencia, 46022 Valencia, Spain. 
frequency ranges in order to have overlapping resonant frequencies. Thus, it is possible to obtain several permittivity values from a resonant method for a wide range of frequencies. Cavities can be designed to have sufficient overlap of resonant frequencies in the permittivity spectrum such that important characteristic behaviors, such as a relaxation process, will not be missed by the measurements.

This work can be applied to characterization of crude oil chemical properties (such as asphaltene or aromatic content) or rheological properties (density) via microwave measurements, providing a faster way to monitor changes that could indicate biodegradation, evaporative fractionation, or thermochemical sulfate reduction in the crude. These processes could be tied to formation quality, and therefore a significant change in electrical properties could indicate a formation damage process [3].

For oil spills, accurate values of oil slick permittivity are needed for proper slick detection and monitoring. In [8], the oil slick from Deepwater Horizon is characterized by fullpolarimetric (FP) synthetic aperture radar (SAR). The authors also showed that the oil was mixed with the sea water in the upper layer of the ocean surface, a mixed layer, with a thickness of 7-9 mm. A mixed layer is a water-in-oil emulsion that is stabilized by the formation of high-strength viscoelastic asphaltene films around water droplets in oil. Although resins can also form emulsions, they are not as stable as the ones formed by asphaltenes. Thus, the size and stability of the mixed layer can have a strong dependence on the crude oil composition [8].

All recent studies on oil spill detection and monitoring [8-12] rely on the Bragg scattering mechanism, whose scattering coefficients depend on the effective complex permittivity of the oil slick, the mixed layer beneath it, and their respective thicknesses. Consequently, accurate permittivity measurements of the oil slick with its mixed layer are needed for more precise calculation of the Bragg scattering coefficients. Moreover, these measurements must be performed onsite at in-situ temperatures.

Due to the compact size of many Vector Network Analyzers (VNA) and of the system of multipoint re-entrant cavities, the system presented in this work is portable. Therefore crude oil measurements can be performed in a trailer at the oil field or in a boat at the site of an oil spill.

The proposed cavities consist of a re-entrant cavity, as shown in Fig. 1, with an insertion hole on the top of the cavity to introduce the samples contained in quartz vials. The quartz vials are the most appropriate as they ensure the tube permittivity remains constant even at the high temperatures of the samples inside.

The insertion hole is below the cut-off frequency to ensure that there is no radiation of the electromagnetic field. Also, there is an insertion hole in the inner conductor of the coaxial, where the bottom section of the tube rests, such that the bottom of the tube is in an area without propagation. This eliminates the effect of the bottom contour, which is difficult to account for in the inversion algorithm.

The proposed analysis method is the Circuit Analysis method, presented in [13] and a summary of which is presented in section II.

\section{THEORY}

The resonance condition in the cavity is analyzed by the equivalent circuit of the cavity [13-17]. In the analysis, the original cavity is divided into smaller segments which can be characterized by the GAM (Generalized Admittance Matrix) [14].

Fig. 1 shows the geometry of a reentrant coaxial cavity, where $b_{2}$ is the coaxial inner radius, $b_{1}$ the coaxial outer radius, $h_{1}$ the coaxial length, and $h_{3}$ the length of circular waveguide. There is one insertion hole on the top of the cavity, of length $h_{4}$, that is below the cut-off frequency in order to avoid leakage of the electromagnetic energy, and a reentrant hole in the inner coaxial conductor, of length $h_{2}$, that is also below the cut-off frequency to avoid problems associated with the non-flat bottom of the tube.

The cavity is fed by a coaxial line through the lateral wall, at height $h_{6}$ and penetration of $h_{5}$. The inserted tube has an external radius of $b_{3}$, and the inner radius is $b_{4}$. The permittivity and permeability of the tube are called $\varepsilon_{r t}$ and $\mu_{r t}$ respectively, and the permittivity and permeability of the sample inside the tube are called $\varepsilon_{r s}$ and $\mu_{r s}$, respectively.

The insertion hole enables the introduction of the sample inside the tube from the upper part and is designed, as mentioned above, to avoid propagation along the hole. Refer to Appendix A for detailed explanation.

Analysis of the cavity is done utilizing the circuit method, described in [15]. The idea behind this method is to divide the whole structure into the smallest and simplest structures that are characterized by their GAM. The smallest structures are then joined together, enforcing the boundary conditions, as performed using the circuit theory. 


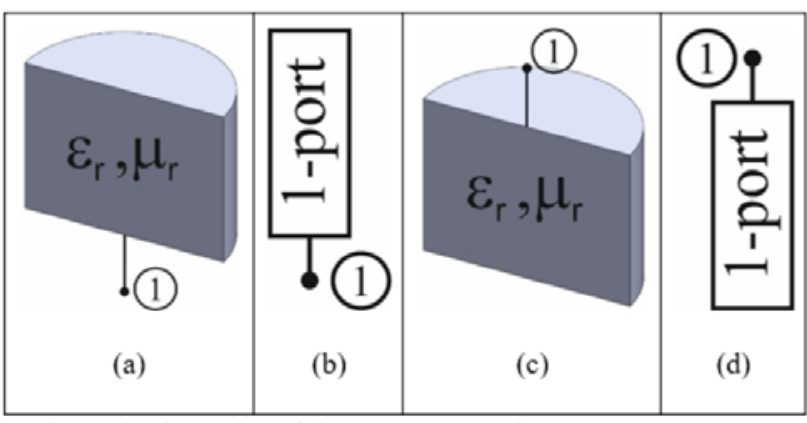

Fig. 2: a) Geometry of the 1-port network (port in the lower side); b) Schematic of the 1-port network (port in the lower side); c) Geometry of the 1-port network (port in the upper side); d) Schematic of the 1-port network (port in the upper side)

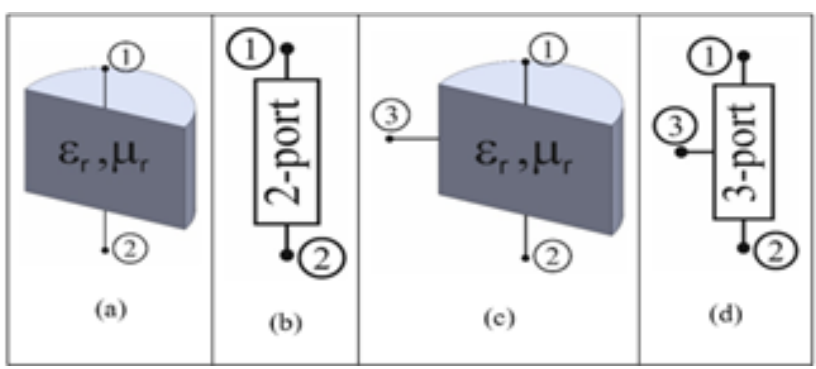

Fig. 3: a) Geometry of the 2-port network; b) Schematic of the 2-port network; c) Geometry of the 3-port network; d) Schematic of the 3-port network

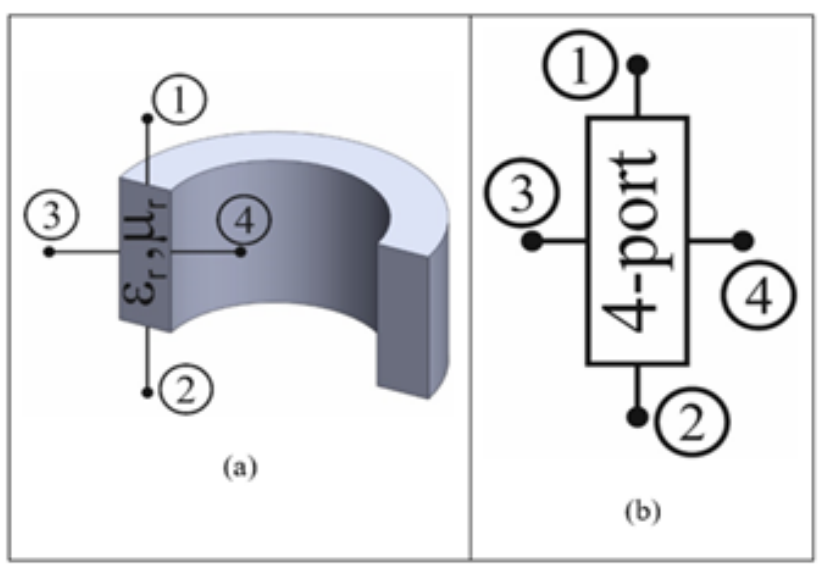

Fig. 4: a) Geometry of the 4-port network; b) Schematic of the 4-port network

In the case of the cavities presented in this work, the structure is segmented into small structures of $1,2,3$, and 4 ports, where each port corresponds to a surface and each network is characterized by its GAM.

These networks are as shown in the next figures. Fig. 2-a is a 1-port network that consists of a cylindrical waveguide with PEC (Perfect Electric Wall) walls in the lateral and top surfaces; the port is the bottom surface. The electric and magnetic fields in this port (or surface) are described by a series expansion, as outlined in [15]. The relationship between the weights of the magnetic and electric series expansions is the GAM [15]. Fig. 2-b is the schematic plot of this 1-port network. It is important to emphasize that this simple model can also be used to model a conductor with finite conductivity, as showed in [14] where the permittivity of the network is modeled with the conductivity of the wall.

Fig. 2-c and 2-d correspond to a 1-port network, but where the port is on the top surface. The 2-port and 3-port networks are the structures shown in Fig. 3-a and 3-c (geometry) and Fig. 3-b and 3-d (schematics). All the above GAM are described in [15].

Finally, the 4-port network is shown in Fig. 4-a (geometry) and 4-b (schematic). The 4-port network GAM is described in [14].

Once the simple geometries are described, it is easy to see how the original geometry (the reentrant cavity shown in Fig. 1 ) is segmented into small structures.

Fig. 5 shows how the original cavity can be segmented into simple 1-port, 2-port, 3-port and 4-port structures.

It is important to reiterate that the top part of the insertion tube does not have any port since the insertion hole is under the cut-off frequency. Thus, there is no propagation and we can consider that there is no reflection from above. 


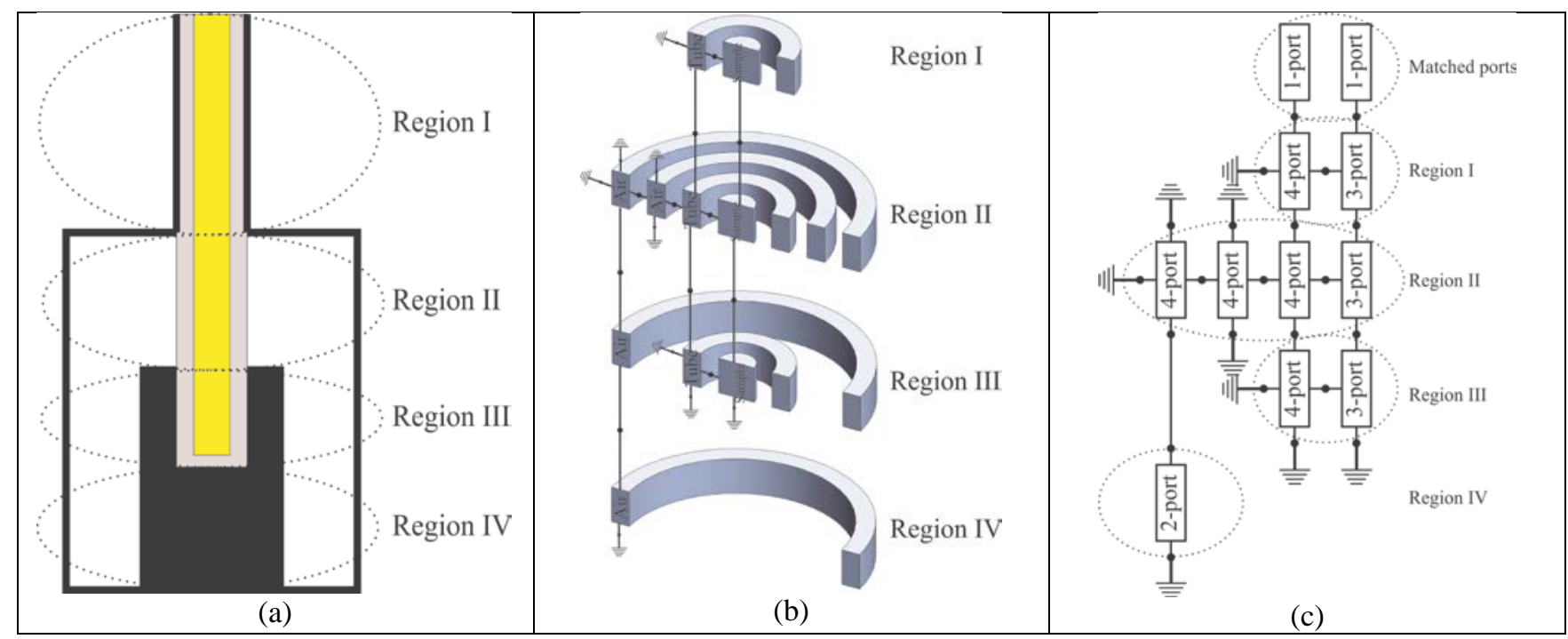

Fig. 5: (a) Geometry of the re-entrant cavity with vials; (b) Segmentation; (c) Schematic

Fig. 5-b shows the segmentation, in each region (from I to IV) with the geometry of the network, and Fig. 5-c the same segmented structure with schematics connected. It must be noted that in the schematics there is a matched port on the top because there is no leakage or propagation through the insertion hole, as previously mentioned.

The theory to connect the common ports is based on Circuit Theory and the boundary conditions between two joined ports. This theory is summarized in Appendix B for a generic case. In the case of the re-entrant cavity, the numbered ports are indicated in Fig. 6-a.

Applying this general theory to our structure, Fig. 6-a shows the general port numbers of the schematic shown in Fig. 5-c.

There are 42 ports to model the whole resonant geometry. If we connect all the networks from port 1 to port 41, leaving alone port 42, we have the geometry shown in Fig. 6-b, where we consider that all the ports inside the shadow are connected (for example ports 33 and 40, 37 and 41, 3 and 5, etc., as explained in Appendix B).

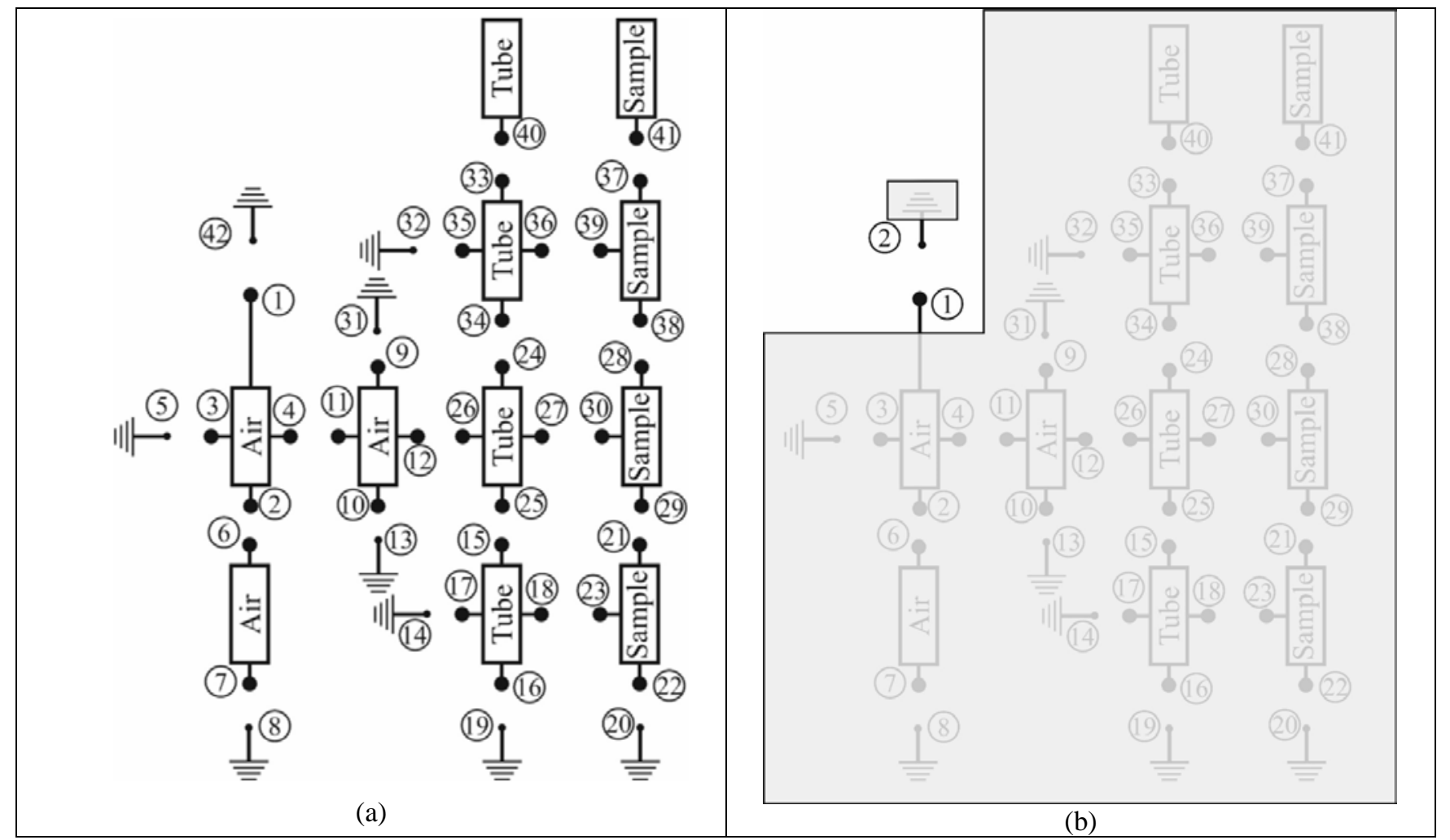

Fig. 6: (a) Schematic with the ports numbered; (b) Resonant geometry selecting only port 1 to port 41, assuming all the ports are connected as explained in Appendix B 


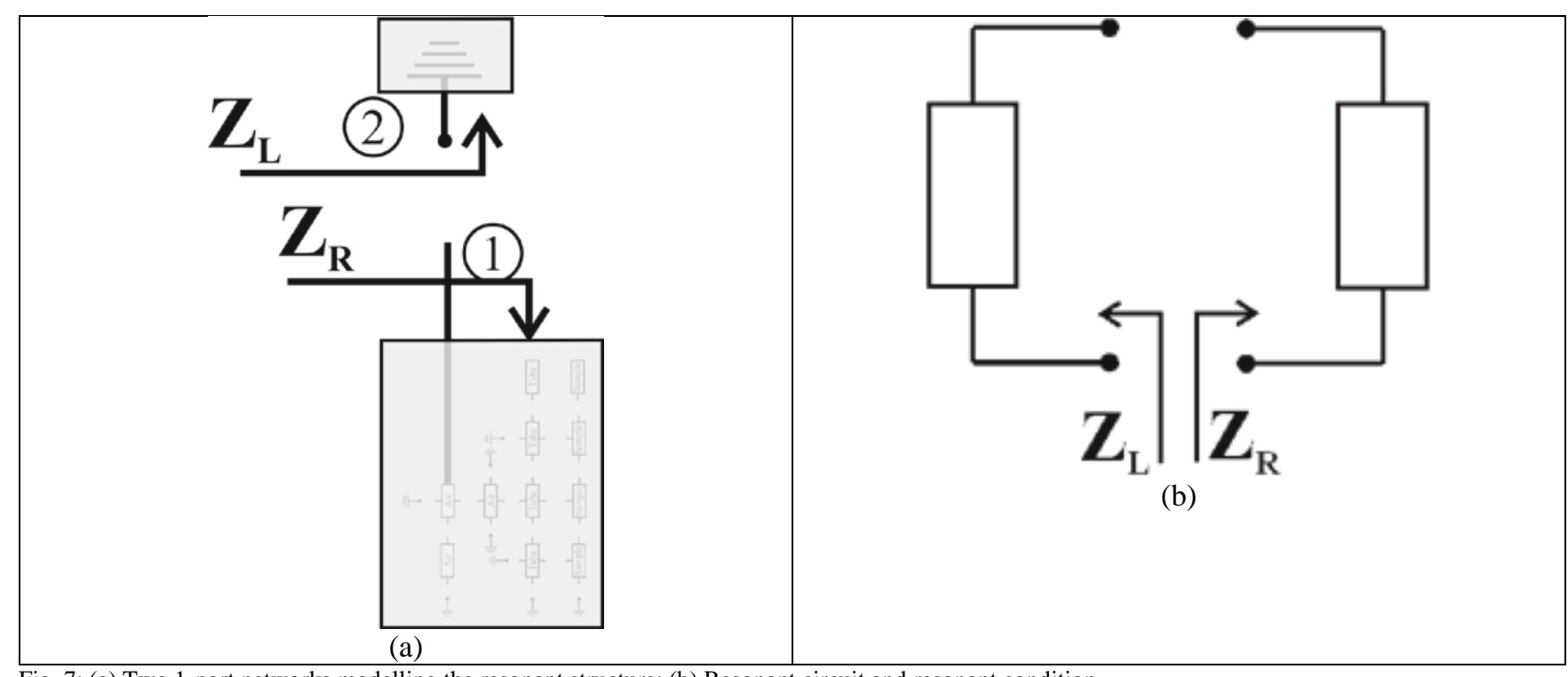

Fig. 7: (a) Two 1-port networks modelling the resonant structure; (b) Resonant circuit and resonant condition

Finally, only two 1-port networks are left, as shown in Fig. 6-b, or Fig. 7-a, both representing the same configuration in which each network is characterized by its own GIM (Generalized Impedance Matrix). It is clear that we can use either GAM (Generalized Admittance Matrix) or GIM (Generalized Impedance Matrix), given their following relationship:

$$
\overline{\bar{Y}}=\overline{\bar{Z}}^{-1}
$$

In fact, when using GIM to characterize the simple elements instead of GAM, the equation to get the final GIM after connecting the common ports is the same as (18) in Appendix B, with $\overline{\bar{Y}}$ replaced by $\overline{\bar{Z}}$.

In Fig. 7-a the GIM is used instead of GAM, because the resonant condition is usually defined with the GIM in this geometry.

In Fig. 7-a, $\overline{\bar{Z}}_{R}$ represents the GIM of all the elements from port 1 to port 41 in Fig. 6-b; $\overline{\bar{Z}}_{L}$ represents the GIM of the element numbered as port 42 in Fig. 6-b and consists of a shortcircuit. The model is shown in $[14,15]$ and includes, as all the 1-port networks that model a short-circuit, the finite conductivity of the conductor, as previously described.

It is clear that the whole resonant geometry can be simplified to a two 1-port networks using the theory described above and calculating the Generalized Admittance Matrices using the expressions of $[14,15]$.

The last step is to calculate the resonant frequency. If the resonant frequency is known because it has been measured, we still need to calculate the permittivity of the sample.

To calculate the resonant frequency, we need to force the resonant condition. This is shown in [13]. Basically, if we have two networks whose GIM are $\overline{\bar{Z}}_{R}$ and $\overline{\bar{Z}}_{L}$, as shown in Fig. 7-b the resonant condition means that:

$$
\overline{\bar{Z}}_{R}+\overline{\bar{Z}}_{L}=\overline{\overline{0}}
$$

Because the GIM are matrices, then, the resonant condition is stated as follows [12]:

$$
\left|\operatorname{det}\left(\overline{\bar{Z}}_{R}+\overline{\bar{Z}}_{L}\right)\right|=0
$$

It is important to remember that GIM and GAM depend on the dimensions, on the electromagnetic properties of the materials and the frequency. In addition, the last matrices involved in the problem to calculate the resonant frequency depend on all these values and equation (5) can be written as:

$$
\left|\operatorname{det}\left(\overline{\bar{Z}}_{R}(\operatorname{dim}, \varepsilon, \mu, f)+\overline{\bar{Z}}_{L}(\operatorname{dim}, \varepsilon, \mu, f)\right)\right|=0
$$

In (6), all the dimensional parameters are known and the resonant frequencies and $\mathrm{Q}$ values are accurately measured by the VNA. The only unknown variable, therefore, is the complex permittivity [7], described in (1).

The permittivity is calculated by solving the transcendental equation (6). In our case, it is solved using the Nelder-Mead simplex (direct search) method.

When the resonant frequency is a complex number, it is called the Complex Resonant Frequency. This is a very important concept because the real part contains information about the resonant frequency and the imaginary part gives information about the quality factor of the cavity. The quality factor is needed to know the losses of the resonant structure.

The definition of the complex resonant frequency can be found in $[18,19]$. The complex resonant frequency, usually written as $\Omega_{r}$, is:

$$
\Omega_{r}=f_{r} \cdot\left(1-\frac{j}{2 Q}\right),
$$

where $f_{r}$ is the resonant frequency and $Q$ is the quality factor of the resonator. The quality factor is associated to the losses and it is defined as:

$$
\mathrm{Q}=\left.2 \pi f \frac{U_{T}}{P_{L}}\right|_{f=f_{r}},
$$


Table I: Dimensions of Cavities

\begin{tabular}{|c|c|c|c|c|c|c|c|c|c|c|}
\hline & \multicolumn{9}{|c|}{ Dimension $[\mathrm{mm}]$} \\
\hline Cavity & $\mathrm{h}_{1}$ & $\mathrm{~h}_{2}$ & $\mathrm{~h}_{3}$ & $\mathrm{~h}_{4}$ & $\mathrm{~h}_{5}$ & $\mathrm{~h}_{6}$ & $\mathrm{~b}_{1}$ & $\mathrm{~b}_{2}$ & $\mathrm{~b}_{3}$ & $\mathrm{~b}_{4}$ \\
\hline 1 & 274 & 20 & 8 & 20 & 2 & 112 & 7 & 7.5 & 6.5 & 5.26 \\
\hline 2 & 29.5 & 20 & 7.7 & 20 & 2 & 36 & 7.03 & 5 & 4.05 & 1.5 \\
\hline
\end{tabular}

where $f_{r}$ is the resonant frequency, $U_{T}$ is the total electric and magnetic energies in the resonator, and $P_{L}$ is the total power dissipated by the resonator $[7,19]$.

The actual dimensions of the cavities are given in Table I, where $h_{5}$ is optimized to couple enough energy to the resonator to couple resonant modes. The tubes are made of quartz, whose permittivity is calculated with the measurements of an empty tube.

As an example of the resonant modes that appear in one of these cavities, the next paragraphs will show the simulations made with the second cavity to see the first $\mathrm{TM}_{0 \mathrm{np}}$ modes that appear in the lower frequencies. It is easy to locate these modes, process, and calculate the permittivity at different resonant frequencies. At higher frequencies, higher modes appear, and it becomes very difficult (or even impossible) to distinguish them from the $\mathrm{TM}_{0 \mathrm{np}}$ modelled.

Fig. 8 shows the first $3 \mathrm{TM}_{\text {onp }}$ resonant modes of cavity 2 simulated with the circuit method describe above, when the permittivity of the tube is $\varepsilon_{\text {rtube }}=3.6-\mathrm{j} \cdot 10^{-4}$ and the sample inside has a permittivity of $\varepsilon_{\mathrm{r}}=5.5-\mathrm{j} \cdot 10^{-2}$.

It is easy to verify that these are the first 3 resonant modes, then they can be measured with a VNA (Vector Network Analyzer), as described below.

The first complex resonant frequency is, in this case:

$$
\Omega_{r}=f_{r} \cdot\left(1-\frac{j}{2 Q}\right)=1.296818 \cdot\left(1-\frac{j}{2 \cdot 7147}\right) \mathrm{GHz}
$$

For the simulation, we assumed that the walls were perfect conductor (PEC).

In summary, the process to calculate the permittivity of the sample consists of measuring different resonant modes with the VNA. The complex resonant frequency is obtained using the above procedure. The resonant condition is then used to calculate the permittivity of the sample.

It is important to mention the way through which the complex resonant frequency (the unloaded resonant frequency and the unloaded quality factor) is obtained. A typical error while performing these kinds of resonant measurements confuses the loaded and unloaded values. The loaded values are the values measured directly with the VNA (the $S_{11}$ measurements), including the effect of the excitation network and the source. The unloaded values are the values with the effect of the excitation network and the source removed.

The process to extract the unloaded information from the actual loaded measurements is complex and described in detail in [20].

Finally, it is interesting to compare these results (we will compare only the computed results) with commercial software.

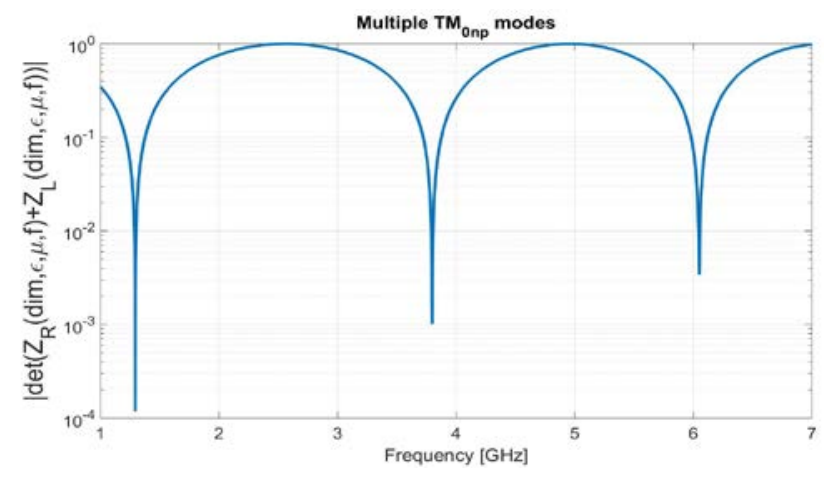

Fig. 8: Simulation of the 3 resonant modes of small cavity

We compared the resonant frequency with the resonant frequency from the software QWED [21] (based on FDTD). The resonant frequency computed with QWED is $f_{r}=$ $1.29757 \cdot\left(1-\frac{j}{2 \cdot 7226}\right) \mathrm{GHz}$. Thus showing a close match with the values in (9).

The magnitude of the electric and magnetic field are plotted in Fig. 9-a and Fig. 9-b respectively. From Fig. 9-a and 9-b, the assumption that there is no propagation along the upper and lower insertion holes is validated. Therefore, the resonant modes are under the cut-off frequency.

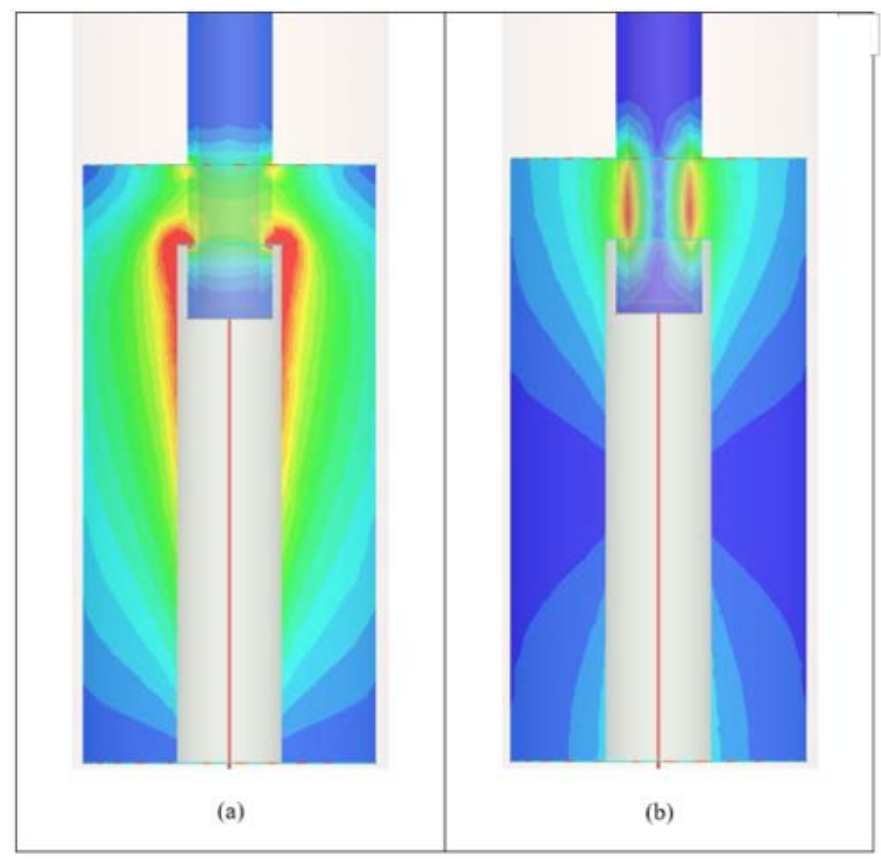

Fig. 9: Normalized modulus of the Electric (a) and magnetic (b) fields 


\section{SETUP, PROCEDURE AND DESIGN}

The experiment setup consists of a Rohde and Schwarz ZVA50 high end VNA, two Gore high stability VNA cables, a $3.5 \mathrm{~mm}$ Agilent 85052B calibration kit, a laptop computer and two cavities with their respective vials. Experiments were performed inside a Faraday cage to block interference from outside sources. The VNA was placed outside the faraday cage and the cables were allowed to enter the faraday cage through two small orifices. See Fig. 10.

The VNA with the cables connected to its ports was calibrated every day with the $3.5 \mathrm{~mm}$ calibration kit. The VNA cables were connected to the cavities, the cavities were then leveled and calibration measurements with empty vials were performed for each mode. The leveling of the cavities was done to prevent vials from being tilted inside the cavity and thereby, producing poor quality resonances and influence from higher order modes. In addition, a vertically straight vial is the assumption of the inversion algorithm. To avoid having to characterize the vials at different temperatures, quartz vials were chosen given their relatively constant complex permittivity spectrum for temperatures up to $700^{\circ} \mathrm{C}$, as per laboratory measurements.

After the measurements with empty vials were performed and saved, the vials were filled with the material under test (MUT), brought to the desired temperature, and then quickly introduced into the respective cavity. Multiple permittivity measurements were taken for different values of key parameters to be able to estimate the combined standard uncertainty and demonstrate repeatability. After saving the measurements on empty and filled vials, the data were transferred to a laptop computer and inverted as described in Section II. Measurements on toluene were performed for validation of the actual measurements. The final uncertainty value is given as an expanded uncertainty with a $\mathrm{k}=2$ coverage factor.

Two cavities were designed to cover a frequency range from $170 \mathrm{MHz}$ to $8.6 \mathrm{GHz}$. Both cavities are cylindrical and

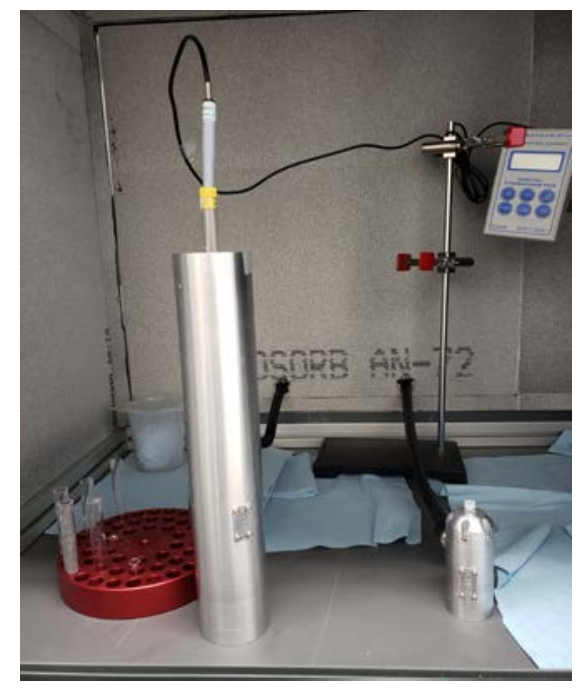

Fig. 10: Both cavities inside the Faraday cage. Vials with caps placed in cavities and temperature probe inserted through cap in large cavity

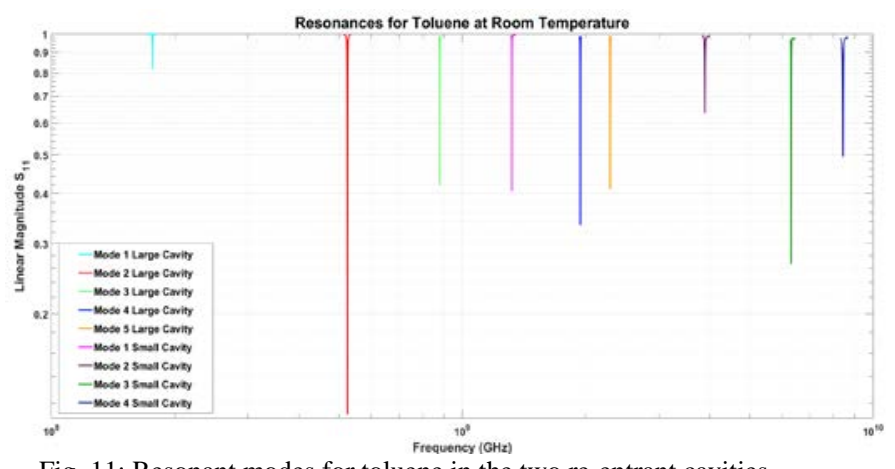

Fig. 11: Resonant modes for toluene in the two re-entrant cavities

designed to hold a vial with liquid or powdered/machined material. The large cavity and small cavity dimensions are given in Table I, Fig. 10 shows both cavities for comparison.

There are nine possible resonant frequencies given by both cavities. The large cavity has five resonant frequencies and the small one has four. The first resonant mode of the small cavity ( $\sim 1.3 \mathrm{GHz})$ is between the third mode $(\sim 0.9 \mathrm{GHz})$ and fourth mode $(\sim 1.9 \mathrm{GHz})$ of the large cavity. This overlap allows verification permittivity results to identify potential issues.

The cavities are made of aluminum, which has an electrical conductivity of $3.54 \times 10^{7} \mathrm{~S} / \mathrm{m}$. In addition, the small cavity has all the inside void spaces filled with air, while the large cavity is filled with Teflon to lower the first resonant frequency. This allows the large cavity to have the first resonant mode around $170 \mathrm{MHz}$. The resonator excitation was placed in an optimal location, based on the electric field distribution inside of the cavity, to maximize the excitation of the TEM mode.

\section{VALIDATION}

Validation measurements were conducted on ACS certified toluene, a pure aromatic compound commonly found in crude oil. Permittivity measurements with toluene at room temperature were conducted and compared with values 


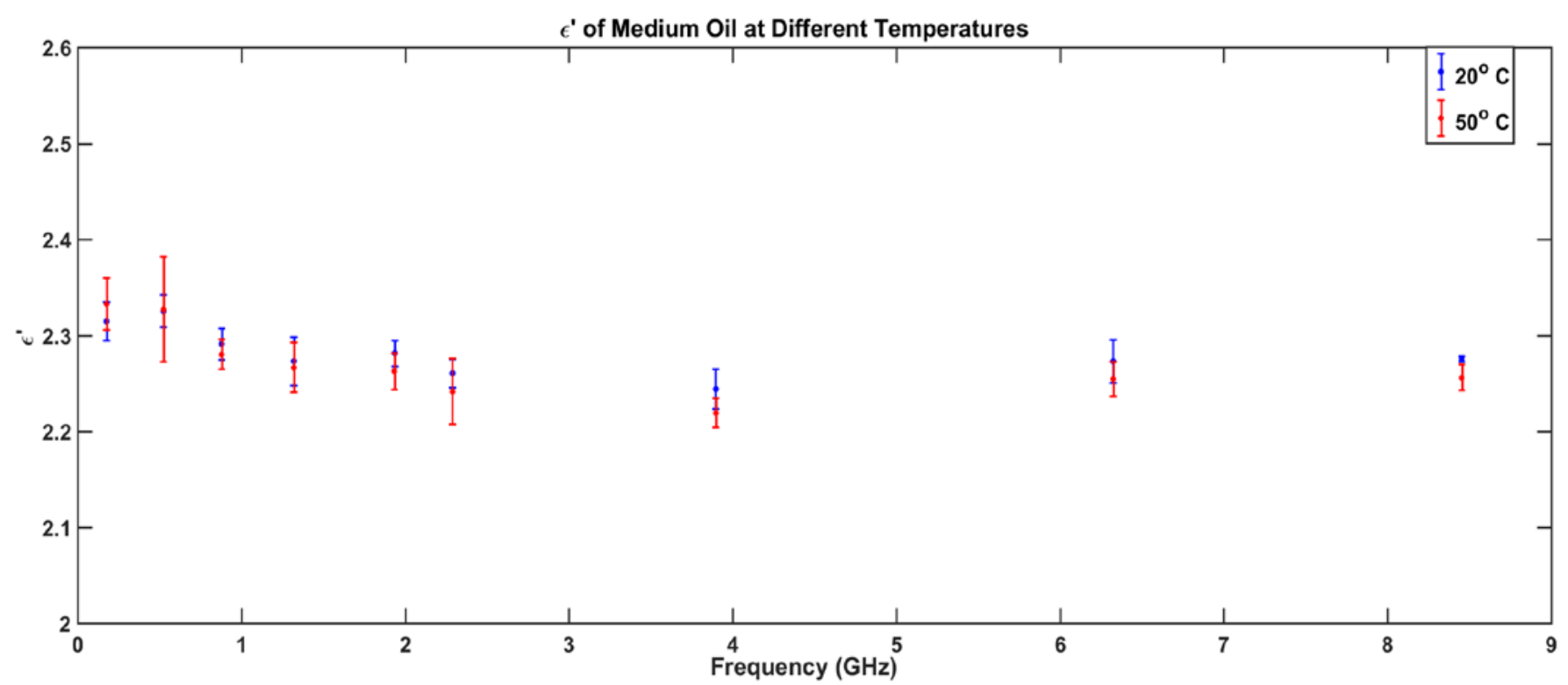

Fig. 13: Real part of the permittivity spectra of medium grade (28.2 API) crude oil at $20^{\circ} \mathrm{C}$ (blue circles) and $50^{\circ} \mathrm{C}$ (red squares) . Error bars denote the uncertainty $(\mathrm{k}=2)$.

obtained by Instituto ITACA with single resonance cavities. Fig. 11 shows the linear magnitude of the $S_{11}$ parameter for a toluene measurements performed in both cavities. It shows nine resonant $\mathrm{TM}_{\text {nnp }}$ modes that have not been mixed with higher order modes.

Fig. 12 shows the complex permittivity values of toluene at room temperature, about $21^{\circ} \mathrm{C}$. Solid markers denote the real part of the complex permittivity with their values in the left vertical axes. Hollow markers denote the imaginary part and their values are given in the right vertical axis. The values obtained for toluene with the built cavities show excellent agreement with those obtained by single resonance cavity at the ITACA institute and very small uncertainty even for the small values of the imaginary part. Thus, the re-entrant cavities provide the accuracy of a resonant method but for an extended number of frequencies.

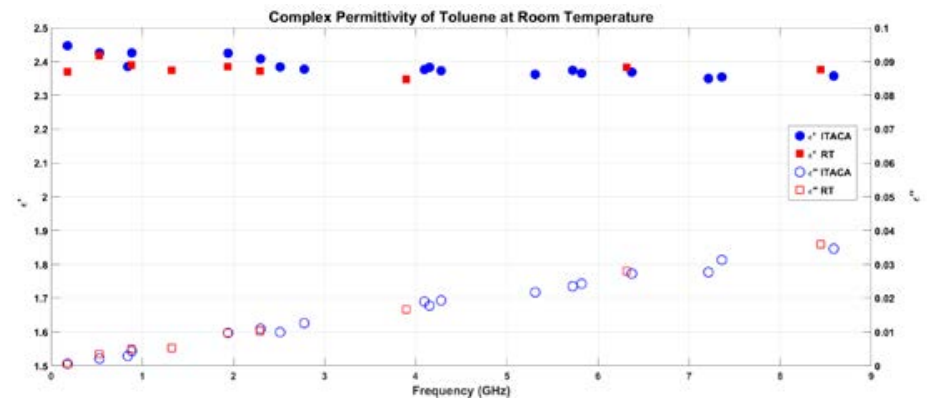

Fig. 12: Complex permittivity spectra of toluene at room temperature $\left(21^{\circ} \mathrm{C}\right)$. Comparison between values obtain with re-entrant cavities. Solid markers denote the real part of the complex permittivity and their values are given in the left vertical axes. Hollow markers denote the imaginary part and their values are given in the right vertical axis.

\section{PermitTivity Spectra}

\section{A. Crude Oil}

Crude oil, like most typical liquid hydrocarbons, consists of a mix of aromatic, saturate, metals, and asphaltene components. Metals are usually present in very small quantities and therefore the aromatics and asphaltenes are the major contributors to losses. Previous work [3-4] showed that the real part can be correlated to the oil density and the losses due to asphaltene content are seen at lower frequencies. Additionally, the aromatic content becomes the major contributor for the losses at higher frequencies. Moreover, toluene and xylenes were shown to be dominant contributors for the imaginary part of the permittivity at high frequencies.

Fig. 13 and 14 show the real and imaginary parts of the complex permittivity of an immature, medium grade (28.2 API) crude oil at $20^{\circ} \mathrm{C}$ and $50^{\circ} \mathrm{C}$. The $20^{\circ} \mathrm{C}$ measurements were taken at $20^{\circ} \mathrm{C}$ and $21.38^{\circ} \mathrm{C}\left(21^{\circ} \mathrm{C}\right.$ for small cavity), and the $50^{\circ}$ $\mathrm{C}$ measurements were taken at $50^{\circ} \mathrm{C}$ and $50.6^{\circ} \mathrm{C}\left(50.4^{\circ} \mathrm{C}\right.$ for small cavity). Higher temperature measurements were taken to obtain temperature sensitivity coefficients to compute temperature uncertainty (section VI). In addition, for both temperatures and cavities, a cap was placed on the vials for temperature stability and to avoid evaporation of lighter aromatic components. In both Fig. 16 and Fig. 17 error bars denote the uncertainty $(\mathrm{k}=2)$. Uncertainty values depend on the mode measured, the vial used, temperature accuracy and stability, slight chemical changes in the samples, as well as other sources. Additional information regarding the uncertainty values is discussed in section VI.

In Fig. 13, the $20^{\circ} \mathrm{C}$ permittivity values are close to those obtain for a similar oil in [3] at $25^{\circ} \mathrm{C}$. At $50^{\circ} \mathrm{C}$, the real part decreases, which is consistent with a decrease in density due to the increase in temperature [3]. In Fig. 14, the imaginary part for both temperatures decreases with frequency and the losses 


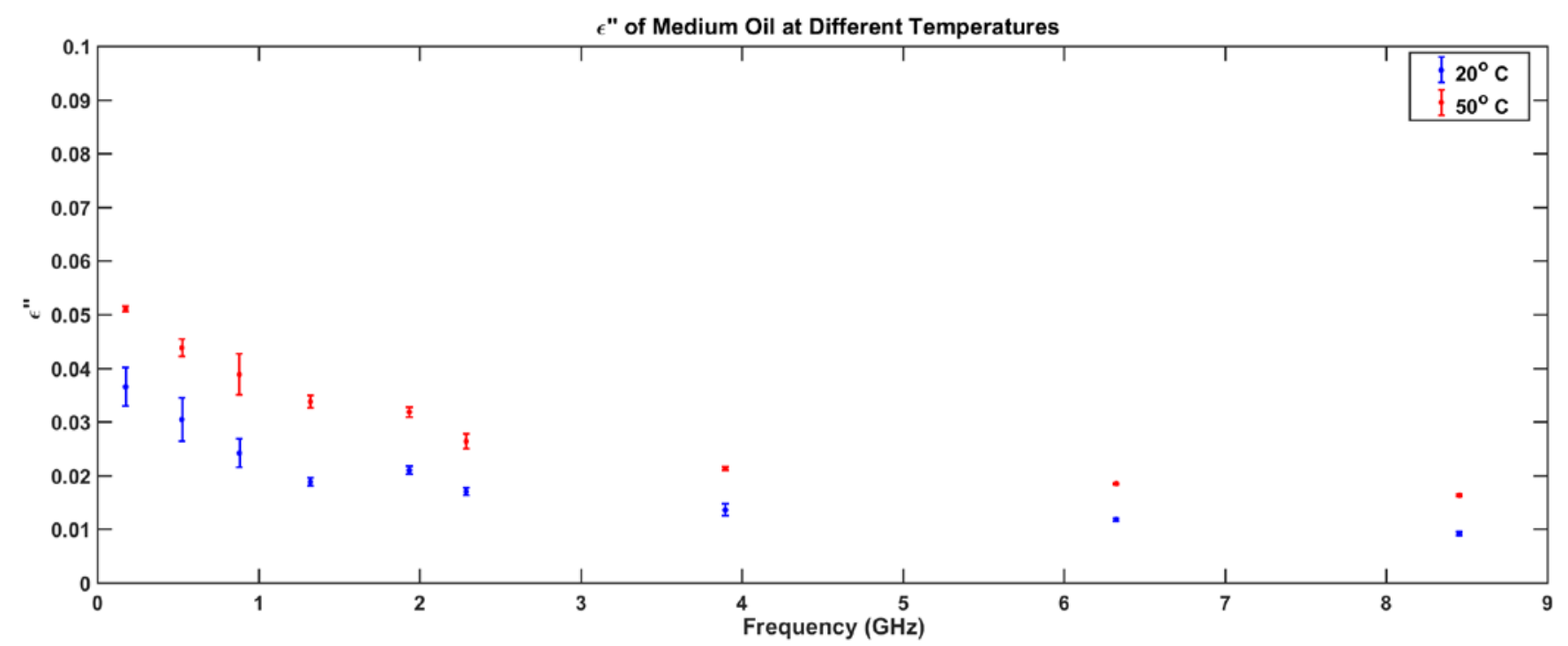

Fig. 14: Imaginary part of the permittivity spectra of medium grade (28.2 API) crude oil at $20^{\circ} \mathrm{C}$ (blue circles) and $50^{\circ} \mathrm{C}$ (red squares). Error bars denote the uncertainty $(\mathrm{k}=2)$.

increase with temperature. There is also a consistent peak around $2 \mathrm{GHz}$ for both temperatures that may be a true relaxation process, although this result needs to be verified by other resonant measurements. Table II shows the values of the measured permittivity and their respective expanded uncertainties

\section{B. Kerogen}

Powders have pore spaces that may or may not affect complex permittivity measurements. How much porosity affects microwave measurements depends on the overall porosity, the pore size, the fluids contained in the pore space, and the frequencies of interest.

Complex permittivity can be obtained by either compensating the measurements for the effect of porosity or by compacting the powder to significantly reduce the porosity and therefore its effect on the measurements. In this study, we performed the latter by compacting the powders inside the quartz vials with heavy tungsten rods to achieve a uniform compaction. The powders were poured into the vials in small stages, to about 1-1.5 vial-diameters in height and then compacted with tungsten rods with similar diameter as the respective vial. Successive stages were added following the same procedure until the desired height was achieved. This guaranteed uniform compacting pressure (16,280 $\mathrm{Pa}$ and 10,822 $\mathrm{Pa}$ in large and small vials, respectively) and eliminated any significant porosity effect.

Kerogen is another geomaterial that, to our knowledge, has not yet had its complex permittivity fully studied. Kerogen, a fraction of sedimentary organic matter, is insoluble in the usual organic solvents. Kerogen distinguishes from bitumen, which is the soluble fraction in the sedimentary organic matter and is mostly made of carbon $(\mathrm{C})$, hydrogen $(\mathrm{H})$, and oxygen $(\mathrm{O})$. The ratios $\mathrm{O} / \mathrm{C}$ and $\mathrm{H} / \mathrm{C}$ are commonly used for elemental analysis of kerogen in form of diagrams (Van Krevelen). There is little difference in the kerogen elemental analysis before and after extraction, since kerogen is the main component of the organic matter. However, kerogen extraction produces oxidation by increasing the $\mathrm{O} / \mathrm{C}$ ratio and decreasing the $\mathrm{H} / \mathrm{C}$ ratio [22].

In general, kerogen burial depth is characterized by a shift from high $\mathrm{H} / \mathrm{C}$ or $\mathrm{O} / \mathrm{C}$ values (low burial depth) to low values of $\mathrm{H} / \mathrm{C}$ and $\mathrm{O} / \mathrm{C}$. There are different evolution paths in the Van Krevelen diagram depending on the type the kerogen, see Fig. 18. These burial evolution paths are also an indication of maturity. In the Van Krevelen diagram, all types of kerogen

Table II

Measured complex permittivity values and their estimated expanded uncertainty $(k=2)$ for

medium crude oil at $20^{\circ} \mathrm{C}$ and $50^{\circ} \mathrm{C}$ (Modes 1-5 = large cavity; Modes 6-9 = small cavity)

\begin{tabular}{|c|c|c|c|c|c|c|c|c|c|c|}
\hline $\begin{array}{c}\text { Medium } \\
\text { Oil }\end{array}$ & $\begin{array}{c}\mathrm{f}(\mathrm{GHz}) \\
20^{\circ} \mathrm{C}\end{array}$ & $\begin{array}{c}\varepsilon^{\prime} \\
20^{\circ} \mathrm{C}\end{array}$ & $\begin{array}{c}\mathrm{U}\left(\varepsilon^{\prime}\right)(\mathrm{k}=2) \\
20^{\circ} \mathrm{C}\end{array}$ & $\begin{array}{c}\varepsilon^{\prime \prime} \\
20^{\circ} \mathrm{C}\end{array}$ & $\begin{array}{c}\mathrm{U}\left(\varepsilon^{\prime \prime}\right)(\mathrm{k}=2) \\
20^{\circ} \mathrm{C}\end{array}$ & $\begin{array}{c}\mathrm{f}(\mathrm{GHz}) \\
50^{\circ} \mathrm{C}\end{array}$ & $\begin{array}{c}\varepsilon^{\prime} \\
50^{\circ} \mathrm{C}\end{array}$ & $\begin{array}{c}\mathrm{U}\left(\varepsilon^{\prime}\right)(\mathrm{k}=2) \\
50^{\circ} \mathrm{C}\end{array}$ & $\begin{array}{c}\varepsilon^{\prime \prime} \\
50^{\circ} \mathrm{C}\end{array}$ & $\begin{array}{c}\mathrm{U}\left(\varepsilon^{\prime \prime}\right)(\mathrm{k}=2) \\
50^{\circ} \mathrm{C}\end{array}$ \\
\hline Mode 1 & 0.1759 & 2.3143 & 0.0200 & 0.0365 & 0.0036 & 0.1759 & 2.3325 & 0.0270 & 0.0510 & $5.10 \mathrm{e}-4$ \\
\hline Mode 2 & 0.5252 & 2.3252 & 0.0170 & 0.0304 & 0.0041 & 0.5251 & 2.3269 & 0.0545 & 0.0438 & 0.0017 \\
\hline Mode 3 & 0.8811 & 2.2907 & 0.0162 & 0.0242 & 0.0026 & 0.8808 & 2.2802 & 0.0157 & 0.0389 & 0.0038 \\
\hline Mode 4 & 1.9362 & 2.2812 & 0.0136 & 0.0210 & $7.70 \mathrm{e}-4$ & 1.9360 & 2.2624 & 0.0191 & 0.0318 & 0.0009 \\
\hline Mode 5 & 2.2893 & 2.2604 & 0.0150 & 0.0170 & $7.10 \mathrm{e}-4$ & 2.2889 & 2.2414 & 0.0342 & 0.0264 & 0.0014 \\
\hline Mode 6 & 1.3210 & 2.2727 & 0.0250 & 0.0188 & $7.2 \mathrm{e}-4$ & 1.3210 & 2.2663 & 0.0259 & 0.0338 & 0.0012 \\
\hline Mode 7 & 3.8970 & 2.2440 & 0.0210 & 0.0136 & 0.0011 & 3.8975 & 2.2191 & 0.0152 & 0.0214 & $3.40 \mathrm{e}-4$ \\
\hline Mode 8 & 6.3221 & 2.2727 & 0.0223 & 0.0118 & $3.2 \mathrm{e}-4$ & 6.3242 & 2.2543 & 0.0182 & 0.0185 & $1.80 \mathrm{e}-4$ \\
\hline Mode 9 & 8.4533 & 2.2750 & 0.0003 & 0.0092 & $3.4 \mathrm{e}-4$ & 8.4543 & 2.2560 & 0.0134 & 0.0163 & $1.90 \mathrm{e}-4$ \\
\hline
\end{tabular}


tend to a common zone as the $\mathrm{H} / \mathrm{C}$ and $\mathrm{O} / \mathrm{C}$ ratios decrease. Thus, maturation produces carbon enrichment in the solid part of the organic matter by the production of products that are richer in oxygen and hydrogen than the original organic matter. Moreover, the quantity of hydrocarbon which will be created during burial can be related to the position in the Van Krevelen diagram [22], see Fig. 15.

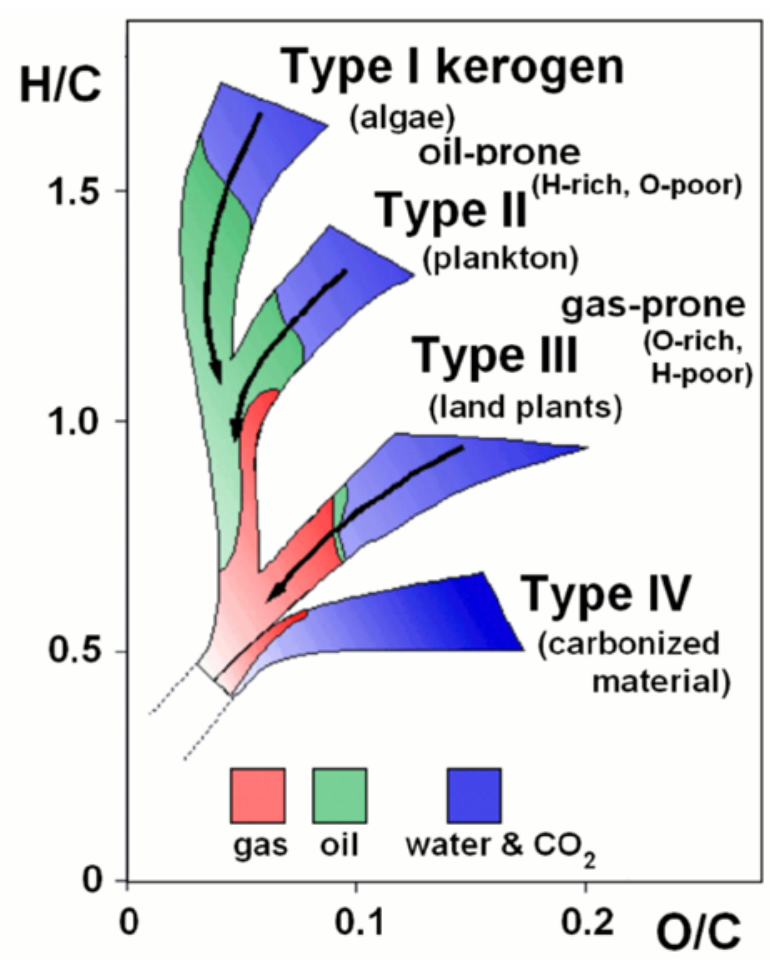

Fig. 15: Van Krevelen diagram from [23]. The maturation paths (from immature to mature) for four types of kerogen are shown as function of $\mathrm{H} / \mathrm{C}$ to $\mathrm{O} / \mathrm{C}$ ratios and its main composition.

As kerogen matures, it produces methane that can remain as gas or be dissolved in water inside the kerogen pores [24]. In addition, water can also be present on the kerogen surface. The degree of the surface wettability (hydrophilicity) is dependent on the organic matter that contains oxygen functional (aliphatic) groups. Mature kerogen will have a higher proportion of hydrophobic to hydrophilic surfaces. From Fig. 15 , the lower maturity kerogen may contain higher $\mathrm{O} / \mathrm{C}$ ratios, which increases the proportion of hydrophilic surfaces [25]. In addition, water isotherms are used to show the difference between hydrophobic coal and hydrophilic shale [24].

Fig.16 shows the complex permittivity values of compacted powdered kerogen measured at room temperature. The kerogen used in the measurements has a vitrinite reflectance of 0.6 , which puts it in the immature kerogen category.

From the above paragraphs it is easy to see that the immature kerogen has a higher ratio of hydrophilic surfaces. Thus, the kerogen sample we measured was allowed to be in contact with air humidity for 24 hours to get as close to saturation as possible. The sample was then compacted in the vial and a cap was put on the vial to avoid any extra water absorption. After that, its temperature was allowed to stabilize to room temperature $\left(20.1^{\circ} \mathrm{C}\right)$.

The real part of the complex permittivity is given in Fig. 16 by blue markers. The real part shows a slow decay from values around 2.5 to values near 2 which is consistent with trends obtained for crude oil and gas condensate $[1,3]$. The imaginary part is given in Fig. 16 by red markers. It also decays from values around 0.5 to 0.2 . For both real and imaginary parts there are two slightly different trends between the large and small cavity modes. This difference could have several sources, ranging from the non-homogeneity of the samples to the random choice of vial used, see section VI. In addition, mode 1 has a lower than recommended quality factor, which increases the uncertainty. Table III shows the measured complex permittivity values and their respective expanded uncertainties.

\section{Iron Disulfide: Pyrite}

Sulfur is a common element whose sedimentary geochemistry involves oxidation-reduction reactions. One of

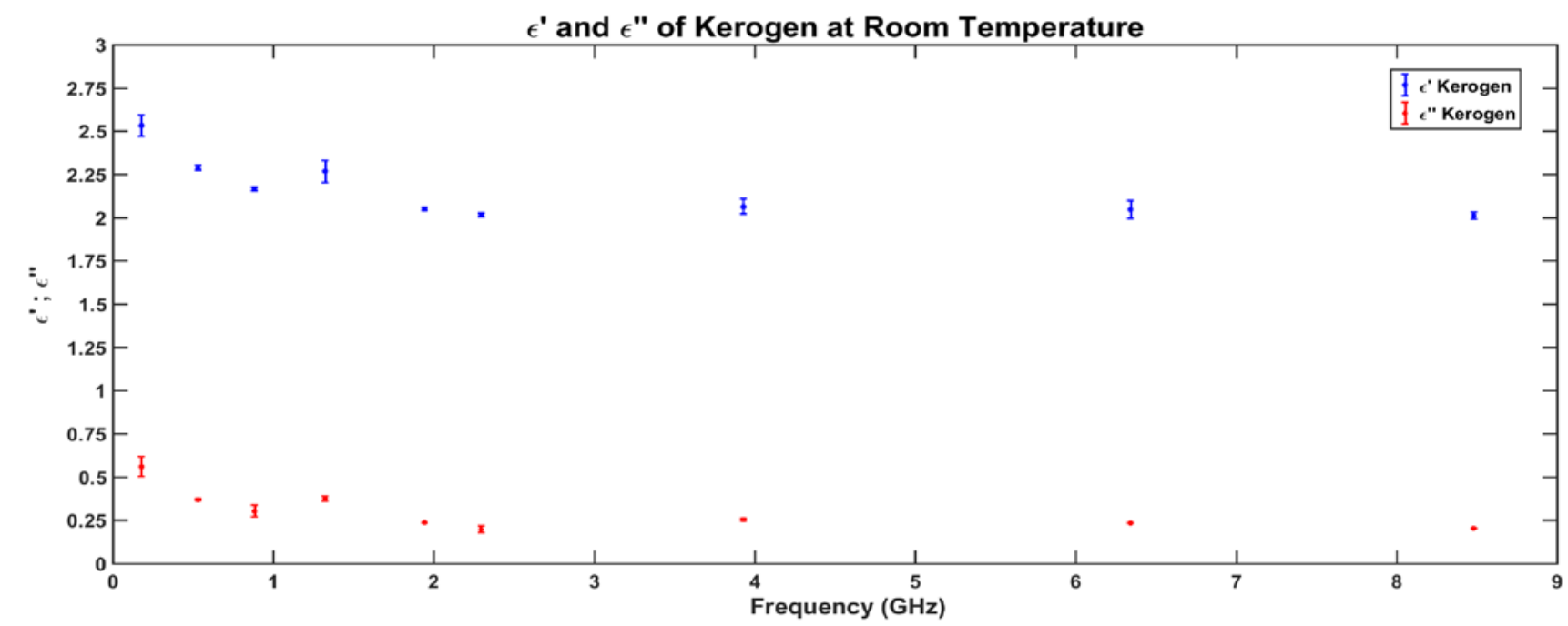

Fig. 16: Real and imaginary part of the complex permittivity spectra of immature kerogen at room temperature $\left.\left(\sim 20.5^{\circ} \mathrm{C}\right)\right)$. Error bars denote the uncertainty $(\mathrm{k}=2)$. 
Table III

Measured complex permittivity values and their estimated expanded uncertainty $(\mathrm{k}=2)$ for immature kerogen

\begin{tabular}{|l|c|c|c|c|c|}
\hline Kerogen & $\begin{array}{c}\mathrm{f} \\
(\mathrm{GHz})\end{array}$ & $\varepsilon^{\prime}$ & $\begin{array}{c}\mathrm{U}\left(\varepsilon^{\prime}\right) \\
(\mathrm{k}=2)\end{array}$ & $\varepsilon^{\prime \prime}$ & $\begin{array}{c}\mathrm{U}\left(\varepsilon^{\prime \prime}\right) \\
(\mathrm{k}=2)\end{array}$ \\
\hline Mode 1 & 0.1762 & 2.5321 & 0.0605 & 0.5607 & 0.0566 \\
\hline Mode 2 & 0.5297 & 2.2887 & 0.0154 & 0.3679 & 0.0046 \\
\hline Mode 3 & 0.8832 & 2.1663 & 0.0114 & 0.3032 & 0.0328 \\
\hline Mode 4 & 1.9423 & 2.0490 & 0.0093 & 0.2364 & 0.0016 \\
\hline Mode 5 & 2.2951 & 2.0153 & 0.0111 & 0.1975 & 0.0204 \\
\hline Mode 6 & 1.3235 & 2.2669 & 0.0629 & 0.3743 & 0.0150 \\
\hline Mode 7 & 3.9280 & 2.0639 & 0.0430 & 0.2536 & 0.0060 \\
\hline Mode 8 & 6.3417 & 2.0461 & 0.0518 & 0.2343 & 0.0030 \\
\hline Mode 9 & 8.4821 & 2.0100 & 0.0185 & 0.2026 & $6.96 \mathrm{e}-4$ \\
\hline
\end{tabular}

the most common oxidation states of sulfur in sedimentary environments are sulfides and the most common sedimentary sulfide is the disulfide iron $\left(\mathrm{FeS}_{2}\right)$ in the form of pyrite or marcasite. Both minerals are found in coal beds, black shales, and bituminous sandstones [26].

In shales, pyrite is believed to have high conductivity that can cause misdiagnosis from the resistivity and dielectric well logs. Thus, the readings can be attributable to water-in-place which will suggest low to no hydrocarbon present and/or be hard to break by hydraulic fracturing (high shale ductility). This can cause well operators to skip producing intervals or to walk away from profitable plays.

The first step towards understanding the true effect of pyrite in electromagnetic readings is to accurately measure its complex permittivity. To our surprise, some clean resonance readings were obtained when compacted disulfide iron powder was measured in the re-entrant cavities. Fig. 17 shows four frequencies that produced readings with most $\mathrm{Q}$ factors around 150. At frequencies between $520 \mathrm{MHz}$ and $2.26 \mathrm{GHz}$ the real part of the permittivity decreases from 13.62 to 11.54 and the imaginary part values show a constant value around 3.7. Although the losses are significant, they are not as high as water with a relative low salinity of $0.1 \mathrm{Mol} / \mathrm{L}(5.8 \mathrm{ppk})$ at $25^{\circ} \mathrm{C}$, whose values are around 20. Thus interfacial polarization effects (Maxwell-Wagner) due to the presence of pyrite will only be noticeable when the pyrite concentration is large enough and its conductivity is much larger (conductive inclusion) or smaller (insulating inclusion) than that of the rest of the medium containing the pyrite inclusions. To add to the complexity, in shales the medium will also contain some percentage of clays, which on their own can have the properties to produce interfacial polarization effects. Much work needs to
Table IV

Measured complex permittivity values and their

estimated expanded uncertainty ( $\mathrm{k}=2$ ) for pyrite

\begin{tabular}{|c|c|c|c|c|c|}
\hline Pyrite & $\begin{array}{c}\mathrm{f} \\
(\mathrm{GHz})\end{array}$ & $\varepsilon^{\prime}$ & $\begin{array}{c}\mathrm{U}\left(\varepsilon^{\prime}\right) \\
(\mathrm{k}=2)\end{array}$ & $\varepsilon^{\prime \prime}$ & $\begin{array}{c}\mathrm{U}\left(\varepsilon^{\prime \prime}\right) \\
(\mathrm{k}=2)\end{array}$ \\
\hline Mode 2 & 0.5201 & 13.6839 & 0.2040 & 4.0104 & 0.3309 \\
\hline Mode 3 & 0.8724 & 12.6616 & 0.1200 & 3.4620 & 0.2600 \\
\hline Mode 4 & 1.9140 & 11.5576 & 0.0706 & 3.6884 & 0.2610 \\
\hline Mode 5 & 2.2600 & 11.5418 & 0.0560 & 3.9239 & 0.1434 \\
\hline
\end{tabular}

be done in this direction to understand the readings from the downhole electromagnetic measurements of shales. Table IV shows the measured complex permittivity values and their respective expanded uncertainty for the four measurable modes

\section{UNCERTAINTY ANALYSIS}

To estimate the expanded uncertainty, the combined standard uncertainty needs to be computed first. It is given by the square root of the combined variance

$$
u_{c}^{2}(y)=\sum_{i=1}^{N}\left(\frac{\partial \epsilon}{\partial x_{i}}\right)^{2} u^{2}\left(x_{i}\right),
$$

where $\epsilon$ is the complex permittivity function and $x_{i}$ are the different input that the permittivity output/measurement depends on. The terms $u^{2}\left(x_{i}\right)$ are the standard uncertainty of each input and the partial derivatives are the sensitivity coefficients.

The main inputs that are involved in the accuracy of the measurements in the present study include: The VNA measurement, measured hydrocarbon temperature, temperature drop of the hydrocarbons while performing the measurement, and vial/cavity diameter. In addition, interference from external sources can be significant in cases where the uncertainty is high.

Uncertainty arriving from the equipment that measures the $S_{11}$ parameter was reduced using a high end Rohde and Schwarz ZVA50 Vector Network Analyzer (VNA), which was allowed to properly warm up to avoid temperature drift, and by using a well characterized $3.5 \mathrm{~mm}$ calibration kit from Agilent 85052B. The variance from the measurement of the $S_{11}$ parameter for a particular mode is given by

$$
\left(\frac{\partial \epsilon}{\partial\left|S_{11}\right|}\right)^{2} u^{2}\left(\left|S_{11}\right|\right),
$$

where $\left|S_{11}\right|$ is the magnitude of the $S_{11}$ coefficient. The estimated uncertainty values were calculated by performing multiple measurements at fixed temperatures. For resonant methods, the uncertainty depends on the magnitude of the reflection coefficient, which is mode dependent. Lower magnitude resonant modes have a higher uncertainty than those with higher magnitude. Thus a mode-by-mode uncertainty analysis was performed.

Temperature is a significant source of uncertainty when conducting permittivity measurements. To measure the 


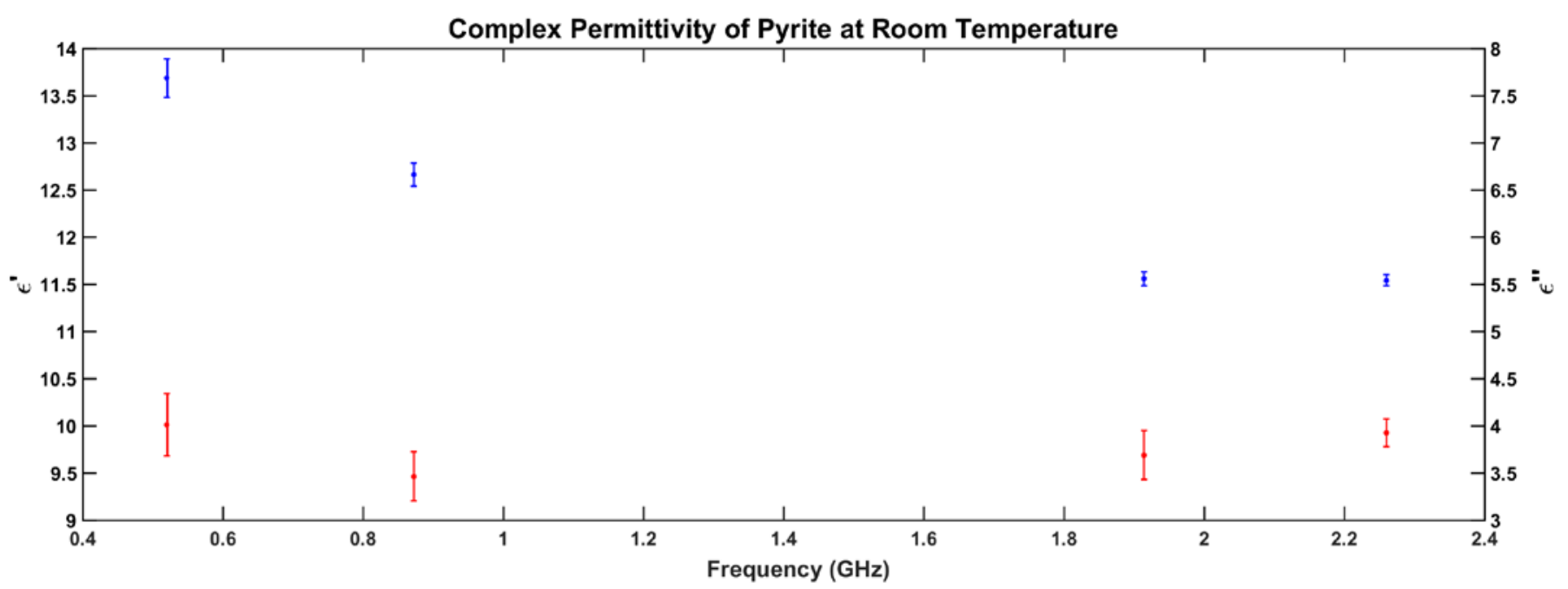

Fig. 17: Complex permittivity spectra of compacted powder pyrite at room temperature $\left(\sim 20.5^{\circ} \mathrm{C}\right)$ (squares). Blue (upper) markers denote the real part of the complex permittivity and their values are given in the left vertical axes. Red (lower) markers denote the imaginary part and their values are given in the right vertical axis. Error bars denote the uncertainty $(\mathrm{k}=2)$.

temperature of the MUT, a NIST calibrated thermometer $(0.01$ ${ }^{\circ} \mathrm{C}$ uncertainty) was used.

The combined variance from the temperature measurements is given by

$$
\left(\frac{\partial \epsilon}{\partial T_{m}}\right)^{2} u^{2}\left(T_{m}\right)+\left(\frac{\partial \epsilon}{\partial T_{m}}\right)^{2} u^{2}\left(T_{t d}\right)
$$

where $T_{m}$ is the measured stable temperature before inserting the vial in the cavity. $T_{t d}$ is the temperature at the actual time of measurement caused by temperature drop due to the low heat storage capacity of hydrocarbons and high temperature gradient. The sensitivity terms are approximated experimentally by performing measurements at different temperatures near the desired temperature, the maximum slope $\frac{\Delta \epsilon}{\Delta T_{m}}$ approximates the sensitivity coefficient.

The uncertainty term $u^{2}\left(T_{m}\right)$ is obtained from the accuracies of the aforementioned thermometers following a similar procedure as in [27]. The uncertainty term $u^{2}\left(T_{t d}\right)$ was calculated experimentally by inserting the vial with MUT and measuring the temperature drop in the time the VNA took to measure the resonance for a particular mode at a particular temperature. Indeed, the higher the measuring temperature gradient with respect to the room/cavity temperature together with a larger VNA measuring times, the higher term $u^{2}\left(T_{t d}\right)$ would be. The measuring time is related to the measuring bandwidth and the width of the resonance of each mode.

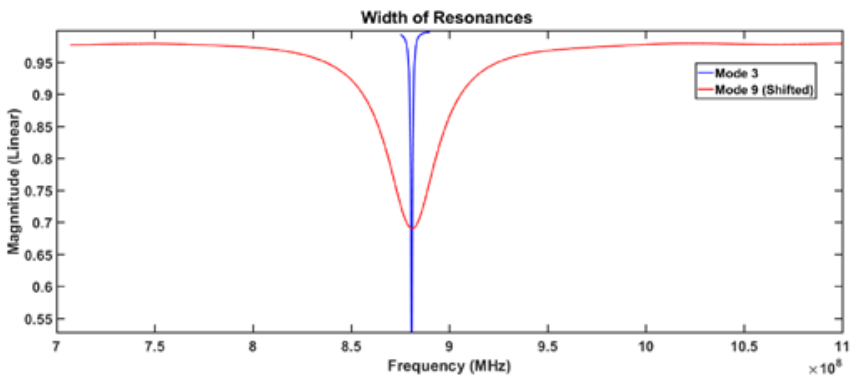

Fig. 18: Linear magnitude of the $S_{11}$ coefficients as a function of frequency for mode 3 and mode 9 (shifted to match).
Indeed, broader measuring bandwidth are inversely proportional to the sweep time. Also, narrower resonances in the magnitude spectrum contain less points than broader resonances, see Fig. 18 . Thus, for wide resonances the measuring bandwidth needs to be increased from $100-200 \mathrm{~Hz}$ to $500 \mathrm{~Hz}$.

In addition, the air conditioning system in the laboratory setting replaces the air in the entire laboratory every four minutes, which produces strong cold air currents that make it difficult to keep high temperatures stable. This had a significant effect on $u^{2}\left(T_{t d}\right)$, which was reduced considerably by placing the cavities and hot plate inside a large Faraday cage with only two opening for the VNA cables.

At $20^{\circ} \mathrm{C}$ and $25^{\circ} \mathrm{C}$, the term $\left(\frac{\partial \epsilon}{\partial T_{m}}\right)^{2} u^{2}\left(T_{t d}\right)$ is negligible, but can be significant at temperatures above $40^{\circ} \mathrm{C}$. For crude oils at $50^{\circ} \mathrm{C}$, the uncertainty $u^{2}\left(T_{t d}\right)$ was measured to be $0.1^{\circ} \mathrm{C}$ for narrow resonances and a maximum of $0.3^{\circ} \mathrm{C}$ for broader resonances.

The uncertainty on the diameter of both the vial and the inside of the cavity can be significant depending on the specific vial diameter tolerance. Although the effect of the vial is somewhat neutralized during calibration with the empty vial, for the inversion algorithm around ten vials within a range of diameters were utilized for the cavity characterization. This has a double effect on the measurements, since the re-entrant cavity algorithm requires calibration with the empty vial that is also used for the MUT. The combined variance from the diameter uncertainty can be estimated by the uncertainty of the measurement with respect to the angle at which the vial is inserted into the cavity, i.e.

$$
\left(\frac{\partial \epsilon}{\partial r o t}\right)^{2} u^{2}(r o t)
$$

In order to estimate the uncertainty term in (13) from the vial position inside the cavity, we first leveled the cavities after connecting them to the VNA cable. Then the vial was marked at a spot and three empty vial measurements are taken at 
Table IV

Relative contribution to the total uncertainty of the quantifiable uncertainties for crude oil at $50^{\circ} \mathrm{C}$

\begin{tabular}{|c|c|c|c|c|c|c|c|c|c|c|}
\hline $\begin{array}{c}\text { Crude Oil } \\
50^{\circ}\end{array}$ & $\begin{array}{c}\mathrm{U}\left(\varepsilon^{\prime}\right) \\
\text { Total }\end{array}$ & $\begin{array}{c}\mathrm{U}\left(\varepsilon^{\prime}\right) \text { Sys } \\
\%\end{array}$ & $\begin{array}{c}\mathrm{U}\left(\varepsilon^{\prime}\right) \operatorname{Rot} \\
\%\end{array}$ & $\begin{array}{c}\mathrm{U}\left(\varepsilon^{\prime}\right) \mathrm{T}_{\mathrm{m}} \\
\%\end{array}$ & $\begin{array}{c}\mathrm{U}\left(\varepsilon^{\prime}\right) \mathrm{T}_{\mathrm{td}} \\
\%\end{array}$ & $\begin{array}{l}U(\varepsilon " ') \\
\text { Total }\end{array}$ & $\begin{array}{c}\mathrm{U}\left(\varepsilon^{\prime \prime}\right) \text { Sys } \\
\%\end{array}$ & $\begin{array}{c}\mathrm{U}\left(\varepsilon^{\prime \prime}\right) \operatorname{Rot} \\
\%\end{array}$ & $\begin{array}{c}\mathrm{U}\left(\varepsilon^{\prime \prime}\right) \mathrm{T}_{\mathrm{m}} \\
\%\end{array}$ & $\begin{array}{c}\mathrm{U}\left(\varepsilon^{\prime \prime}\right) \mathrm{T}_{\mathrm{td}} \\
\%\end{array}$ \\
\hline Mode 1 & 0.0270 & 55.3 & 16.7 & 1.5 & 26.5 & $5.1 \mathrm{e}-4$ & 32.9 & 8.5 & 3.2 & 55.4 \\
\hline Mode 2 & 0.0545 & 39.0 & 11.0 & 1.0 & 49 & 0.00167 & 44.8 & 33.3 & 0.4 & 21.4 \\
\hline Mode 3 & 0.0157 & 50.8 & 20.0 & 1.6 & 27.6 & 0.00380 & 72.1 & 17.1 & 0.1 & 10.7 \\
\hline Mode 4 & 0.0191 & 55.5 & 19.1 & 1.4 & 24.0 & $8.9 e-4$ & 66.2 & 5.3 & 1.6 & 26.9 \\
\hline Mode 5 & 0.0342 & 38.9 & 12.3 & 1 & 47.8 & 0.00140 & 35.8 & 3.3 & 1.2 & 59.7 \\
\hline Mode 6 & 0.0259 & 54.0 & 22.2 & 1.3 & 22.5 & 0.00116 & 63.7 & 4.0 & 1.8 & 30.5 \\
\hline Mode 7 & 0.0152 & 49.8 & 28.0 & 1.2 & 21.0 & $3.4 \mathrm{e}-4$ & 52.0 & 38.0 & 0.5 & 9.5 \\
\hline Mode 8 & 0.0182 & 50.5 & 12.2 & 2.0 & 35.3 & $1.8 \mathrm{e}-4$ & 43.3 & 21.9 & 1.9 & 32.9 \\
\hline Mode 9 & 0.0134 & 32.4 & 27.9 & 0.7 & 39.0 & $1.9 \mathrm{e}-4$ & 29.7 & 21.3 & 1.0 & 48.0 \\
\hline
\end{tabular}

different rotating angles of approximately $\pm 30^{\circ}$ with respect to the marked spot at a fixed point on the surface of the cavity. This is done so that each MUT measurement is compared to three measurements with an empty vial in the inversion process.

In some experiments, the random choice of vial may produce a slight discrepancy between the permittivity trends between cavities. In cases like these, two or three measurements with different vials will give a clearer picture of the vial diameter uncertainty. Given the accuracy of the resonant system and the relatively small percentage difference between different cavity trends at higher frequencies, a fit curve will suffice in most cases to obtain good accuracy values.

Geomaterials present the added complication of being inhomogeneous and/or changing their composition over time. Their effects are easily confused with other sources, which makes the uncertainty from these factors difficult to quantify. In both cases, repeatability is not an issue since in a short amount of time the properties vary within an acceptable uncertainty, if proper precautions are taken. However, reproducibility [27] may become an issue that has to be surpassed by performing many more experiments with the same geomaterial that has been appropriately preserved. Unfortunately, availability of many geomaterials of interest may be limited.

Inhomogeneity implies that samples from the same batch may produce slightly different results. In particular, values from different cavities may give slightly off the trend values in the overlapping frequencies. This is a similar effect to that of vial diameter uncertainty, hence difficult to separate and quantify. Immature kerogen or powdered shales are examples of inhomogeneous geomaterials. If chemical composition changes with time, similar effects to inhomogeneity can be observed from cavity to cavity. Examples of this include crude oil and kerogen. Crude oil under high temperatures produces evaporation of the lighter aromatic components and immature kerogen tends to be hydrophilic, changing its water content with time. These effects may be minimized by proper sample preservation and preparation. Examples of this include: using caps on the vials to reduce aromatic evaporation or water absorption and letting kerogen sit out in the air for twenty four hours to let it absorb water to saturation levels.

External sources of uncertainty may also affect the accuracy of the measurements and may be confused with other uncertainty sources. An example is the magnetic pulse of the Nuclear Magnetic Resonance equipment (NMR) at $525 \mathrm{MHz}$. This frequency is at the resonant frequency of mode 2 of the large cavity that was already inside the Faraday cage. However, mode 2 has the lowest amplitude/highest uncertainty of all modes, making it sensitive to external sources and therefore producing many incoherent values. This interference was mitigated by shielding the VNA with copper sheets and by increasing the dynamic range for mode 2 by reducing the measuring bandwidth of the lower intermediate frequency (IF) bandpass filter $\left(\mathrm{B}_{\mathrm{IF}}\right)$. Indeed, the dynamic range is the difference between the VNA maximum input power and the minimum measurable power (noise level). The noise level (in $\mathrm{dB}$ ) is given by [28]

$$
N_{L}=-174+N_{F}+10 \log \left(S_{F}\right)+10 \log \left(\frac{B_{I F}}{1 H z}\right)
$$

From (14) it is easy to see that for a given noise figure and noise factor of the VNA, if the IF bandwidth is decreased by a factor of $10^{\alpha}$, the noise level goes down by $\alpha \times 10 \mathrm{~dB}$. Thus reducing the IF bandwidth at the VNA reduces the noise level, which in turn increases the dynamic range. 
Table V

Relative contribution to the total uncertainty of the quantifiable uncertainties for kerogen at room temperature

\begin{tabular}{|c|c|c|c|c|c|c|}
\hline $\begin{array}{c}\text { Kerogen } \\
\text { Room Temp }\end{array}$ & $\begin{array}{l}\left.\text { U( } \varepsilon^{\prime}\right) \\
\text { Total }\end{array}$ & $\begin{array}{c}\varepsilon^{\prime}\left(\varepsilon^{\prime}\right) \text { Sys } \\
\%\end{array}$ & $\begin{array}{c}\mathrm{U}\left(\varepsilon^{\prime}\right) \text { Rot } \\
\%\end{array}$ & $\begin{array}{l}\mathrm{U}\left(\varepsilon^{\prime \prime}\right) \\
\text { Total }\end{array}$ & $\begin{array}{c}\mathrm{U}(\varepsilon ") \text { Sys } \\
\%\end{array}$ & $\begin{array}{c}\mathrm{U}\left(\varepsilon^{\prime \prime}\right) \text { Rot } \\
\%\end{array}$ \\
\hline Mode 1 & 0.0605 & 7.6 & 92.3 & 0.0566 & 9.5 & 90.5 \\
\hline Mode 2 & 0.0154 & 63.9 & 36.1 & 0.0046 & 92.2 & 7.8 \\
\hline Mode 3 & 0.0114 & 54.4 & 45.6 & 0.0328 & 83.6 & 16.4 \\
\hline Mode 4 & 0.0093 & 57.7 & 42.3 & 0.0016 & 94.4 & 5.6 \\
\hline Mode 5 & 0.0111 & 70.0 & 30.0 & 0.0204 & 97.7 & 2.3 \\
\hline Mode 6 & 0.0629 & 88.9 & 11.1 & 0.0150 & 78.2 & 21.8 \\
\hline Mode 7 & 0.0430 & 86.2 & 13.8 & 0.0060 & 99.5 & 0.5 \\
\hline Mode 8 & 0.0518 & 64.2 & 35.8 & 0.0030 & 93.8 & 6.2 \\
\hline Mode 9 & 0.0185 & $\begin{array}{l}59.8 \\
\end{array}$ & 40.2 & $6.96 \mathrm{e}-4$ & $\begin{array}{l}64.7 \\
\end{array}$ & 35.3 \\
\hline
\end{tabular}

From the above analysis, contributions from each quantifiable uncertainty source can be computed. Moreover, difficult to quantify uncertainty sources can also be identified for each measurement. Tables IV and V show the relative contribution of each quantifiable uncertainty relative to the total quantifiable uncertainty for medium grade crude oil at $50^{\circ} \mathrm{C}$ and for kerogen at room temperature.

As long as the steps above were taken to reduce the total uncertainty, the inversion algorithm presented above did not appear to be a noticeable source of uncertainty. Even with all of the above uncertainties, uncertainty coming from systematic errors could be significant and harder to quantify. For this reason, the measurement obtained with the present devices could be replaced in the future if better measurements become available.

\section{CONCLUSIONS AND FUTURE WORK}

The theory and design of multipoint coaxial re-entrant resonant cavities was presented. Multipoint resonances are achieved from the same re-entrant coaxial cavity using the harmonics $\mathrm{TM}_{0 \text { np }}$ that are not mixed with other higher modes [29-30]. Once these higher modes appear the method is remains valid, but the resonances must be selected very carefully, especially if small changes in the permittivity are expected. For large changes, this method fails unless a continuous monitoring of the resonances is made by introducing the sample slowly.

Two multipoint cavities and software were built and validated. The first cavity has five resonant frequencies from $170 \mathrm{MHz}$ to $2.3 \mathrm{GHz}$ and the second has four resonant frequencies between $1.3 \mathrm{GHz}$ and $8.6 \mathrm{GHz}$. The cavities were tested with toluene and the results compared with those obtained with single resonance cavities, obtaining a very good match. In addition, measurements can be performed at different temperatures by using quartz vials.

Permittivity values of medium grade crude oil at $25^{\circ} \mathrm{C}$ and $50^{\circ} \mathrm{C}$ were obtained. Results show a decay in dielectric constant with temperature increase and a strong effect of volatility of the aromatic component. Measurements were also performed on kerogen and cement powder.
Resonances and permittivity values were obtained for pyrite at four different frequencies. These values show that the interfacial polarization effects in shales attributed to pyrite inclusions need to be revisited and evaluated. Better knowledge of the electrical properties of the other shale components is needed, in particular water formation and clay content.

Future work will focus on collaborations for measuring oil/water from oil spills and hydrocarbons and aromatics at different temperatures. It will also include the measurements on machined rock samples to compare with results obtained with other microwave fixtures [31].

\section{APPENDIX A}

The insertion hole, for the vials, as well as the reentrant hole in the inner conductor has a radius small enough to guarantee that the circular waveguide is under the cut-off frequency [7]:

$$
f_{c}=\frac{k_{c}}{2 \pi \sqrt{\mu \epsilon}}=\frac{k_{c}}{2 \pi \sqrt{\mu \epsilon_{0} \epsilon_{r}}}
$$

where $k_{c}$ is the cut-off wavenumber and it depends on the propagation mode. The lowest mode in a circular waveguide is $\mathrm{TE}_{11}$ and in our case, because of the geometry of the structure, it will be $\mathrm{TM}_{01}$ [7]. For both cases, the cut-off frequency is when the insertion hole radius is $6.5 \mathrm{~mm}$ :

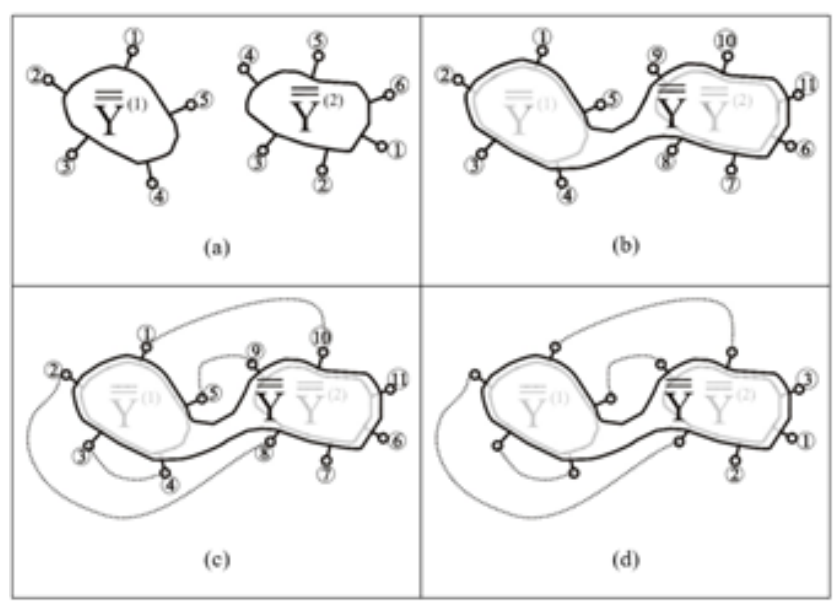

Fig. 19: Circuit Theory applied to N-port networks 


$$
f_{c}=\frac{k_{c}}{2 \pi \sqrt{\mu \epsilon_{0} \epsilon_{r}}}=\left\{\begin{array}{l}
\left.\frac{p^{\prime} 11 / b_{3}}{2 \pi \sqrt{\mu \epsilon_{0} \epsilon_{r}}}\right|_{T E_{11}}=\frac{13.5152 \mathrm{GHz}}{\sqrt{\epsilon_{r}}} \\
\left.\frac{p_{01} / b_{3}}{2 \pi \sqrt{\mu \epsilon_{0} \epsilon_{r}}}\right|_{T M_{01}}=\frac{17.6527 \mathrm{GHz}}{\sqrt{\epsilon_{r}}}
\end{array}\right.
$$

and the cut-off frequency for the high frequency resonant cavity, when the insertion hole radius is $4.05 \mathrm{~mm}$, is:

$$
f_{c}=\frac{k_{c}}{2 \pi \sqrt{\mu \epsilon_{0} \epsilon_{r}}}=\left\{\begin{array}{l}
\left.\frac{p_{11} / b_{3}}{2 \pi \sqrt{\mu \epsilon_{0} \epsilon_{r}}}\right|_{T E_{11}}=\frac{21.6912 \mathrm{GHz}}{\sqrt{\epsilon_{r}}} \\
\left.\frac{p_{01} / b_{3}}{2 \pi \sqrt{\mu \epsilon_{0} \epsilon_{r}}}\right|_{T M_{01}}=\frac{28.3315 \mathrm{GHz}}{\sqrt{\epsilon_{r}}}
\end{array}\right.
$$

The values depend on the permittivity of the sample inside the insertion hole. Because the permittivity of the tube is around 3.5 (it is quartz) and the expected permittivity values of the sample are higher than the quartz one, by using the permittivity of the sample we take into account the worst case to guarantee that there is no propagation along the insertion hole. For example, for values around 10 the cut-off frequencies are, for the large cavity:

$$
f_{c}=\left\{\begin{array}{l}
\left.\frac{13.5152 \mathrm{GHz}}{\sqrt{\epsilon_{r}}}\right|_{T E_{11}}=4.2739 \mathrm{GHz} \\
\left.\frac{17.6527 \mathrm{GHz}}{\sqrt{\epsilon_{r}}}\right|_{T M_{01}}=5.5823 \mathrm{GHz}
\end{array}\right.
$$

and for the same dielectric sample and small resonant cavity, with an insertion hole of $4.05 \mathrm{~mm}$, the cut-off frequencies are:

$$
f_{c}=\frac{k_{c}}{2 \pi \sqrt{\mu \epsilon_{0} \epsilon_{r}}}=\left\{\begin{array}{l}
\left.\frac{p^{\prime}{ }_{11} / b_{3}}{2 \pi \sqrt{\mu \epsilon_{0} \epsilon_{r}}}\right|_{T E_{11}}=6.8593 \mathrm{GHz} \\
\left.\frac{p_{01} / b_{3}}{2 \pi \sqrt{\mu \epsilon_{0} \epsilon_{r}}}\right|_{T M_{01}}=8.9592 \mathrm{GHz}
\end{array}\right.
$$

This means that any signal with higher resonant frequency cannot be used as a resonance because the cavity becomes an antenna and the electromagnetic model does not consider any leakage of the signal.

\section{APPENDIX B}

In this appendix we show how to interconnect different $\mathrm{N}$ port networks forcing the boundary condition in the common ports.

Consider two different networks, for example, of 5 and 6 ports. These networks are characterized by their GAM, called $\overline{\bar{Y}}^{(1)}$ and $\overline{\bar{Y}}^{(2)}$ (see $[14,15]$ to see how these $5 \times 5$ and $6 \times 6$ GAM are constructed). These networks are plotted in Fig. 19-a.

A more general 11-port network can be constructed (see Fig. 19-b), whose GAM is as follows:

$$
\overline{\bar{Y}}=\left(\begin{array}{cc}
\overline{\bar{Y}}^{(1)} & \overline{\overline{0}} \\
\overline{\overline{0}} & \overline{\bar{Y}}^{(2)}
\end{array}\right)
$$

Then, if we want to join together ports 1 and 10, 2 and 8, 3 and 4, and 5 and 9 (as shown in Fig. 19-c, a new GAM is obtained with only 3 ports. In our case, these 3 ports were originally ports 6,7 , and 11 in the 11-port network, but after joining the common ports, these 3 ports are renamed as ports 1,2 , and 3 of the new 3-port GAM (see Fig. 19-d).

The new GAM ( 3 x 3 matrix) can be obtained as follows:

$$
\overline{\bar{Y}}=\left(\begin{array}{lll}
\overline{\bar{Y}}_{11} & \overline{\bar{Y}}_{12} & \overline{\bar{Y}}_{13} \\
\overline{\bar{Y}}_{21} & \overline{\bar{Y}}_{22} & \overline{\bar{Y}}_{23} \\
\overline{\bar{Y}}_{31} & \overline{\bar{Y}}_{32} & \overline{\bar{Y}}_{33}
\end{array}\right)
$$

where each element is calculated with the following recursive general relationship:

$$
\begin{gathered}
\left.\overline{\overline{\mathrm{Y}}}_{\mathrm{pq}}^{(\mathrm{T})}\right|_{\substack{1 \leq \mathrm{p} \leq(\mathrm{N}-2) \\
1 \leq \mathrm{q} \leq(\mathrm{N}-2)}}= \\
\left.\overline{\overline{\mathrm{Y}}}_{\mathrm{pq}}\right|_{\substack{1 \leq \mathrm{p} \leq(\mathrm{N}-2) \\
1 \leq \mathrm{q} \leq(\mathrm{N}-2)}}+ \\
\left(\overline{\overline{\mathrm{Y}}}_{\mathrm{pk}}+\overline{\overline{\mathrm{Y}}}_{\mathrm{pl}}\right) \cdot\left(\overline{\overline{\mathrm{Y}}}_{\mathrm{lk}}+\overline{\overline{\mathrm{Y}}}_{\mathrm{ll}}-\overline{\overline{\mathrm{Y}}}_{\mathrm{kl}}-\overline{\overline{\mathrm{Y}}}_{\mathrm{kk}}\right)^{-1} \cdot\left(\overline{\overline{\mathrm{Y}}}_{\mathrm{kq}}+\overline{\overline{\mathrm{Y}}}_{\mathrm{lq}}\right)
\end{gathered}
$$

$\mathrm{N}$ is the total number of ports of the original network (in our example, $\mathrm{N}=11)$, the pair $(\mathrm{l}, \mathrm{k})$ are the ports that are connected and the pairs $(p, q)$ are the new pairs in the new GAM with $\mathrm{N}-2$ ports in each iteration. In the 11-port example, the number of iterations are 4 , because there are 8 ports connected, having in the end a 3-port network and its GAM of 3x3 dimensions.

\section{ACKNOWLEDGMENT}

J. O. Alvarez thanks David Jacobi for his invaluable teachings and assistance.

\section{REFERENCES}

[1] K. Folgero, "Broad-Band Dielectric Spectroscopy of Low-Permittivity Fluids Using One Measurement Cell,” IEEE Trans. Instrum. Meas., vol. 47, no. 4, pp. 881-885, 1998.

[2] D. Ellis and J. M. Singer, Well Logging for Earth Scientist, 2nd ed., Springer, Dordrecht, The Netherlands, 2008.

[3] J. O. Alvarez, D. Jacobi, and G. Bernero, "Dielectric Characterization of Geochemical Properties of Crude Oils and Gas Condensate at $25^{\circ} \mathrm{C}$," in Proc. International Geoscience and Remote Sensing Symposium (IGARSS), July, 2017, Fort Worth, TX, USA. pp 365-368.

[4] A. Punase, and B. Hascakir, "Stability Determination of Asphaltenes through Dielectric Constant Measurements of Polar Oil Fractions,” Energy \& Fuels, vol. 31, pp. 65-72, 2017.

[5] J. A. Ajienka, N. O. Ogbe, and B. C. Ezeaniekwe, "Measurement of dielectric constant of oilfield emulsions and its application to emulsion resolution,” Jour. Pet. Sci. Eng., vol. 31, pp. 331-339, 1993.

[6] G. Jannin, S. Keffi, and BF. Legendre, "Dielectric Properties of Nonaqueous Drilling Fluids from $1 \mathrm{MHz}$ up to $20 \mathrm{GHz}$," IEEE Trans. Diel. Elec. Ins., vol. 21, no. 1, pp. 217-224, 2014.

[7] Robert E. Collin, "Foundations for Microwave Engineering”, IEEE Press, 2001, ISBN 9780780360310

[8] B. Minchew, C. E. Jones, and B. Holt, "Polarimetric analysis of backscatter from the deepwater horizon oil spill using L-band synthetic aperture radar," IEEE Trans. Geosci. Remote Sens., vol. 50, no. 10, pp. 38123830, Oct. 2012.

[9] M. Fingas and B. Fieldhouse, Handbook of Oil Spill Science and Technology. 1st edition, Hoboken, New Jersey: JohnWiley \& Sons, ch. 8, pp. 230-23, 2015. ISBN: 978-0-470-45551-7

[10] A.-B. Salberg, O. Rudjord, and A. H. S. Solberg, "Oil spill detection in hybrid-polarimetric SAR images,” IEEE Trans. Geosci. Remote Sens., vol. 52, no. 10, pp. 6521-6533, Oct. 2014.

[11] M. M. Espeseth, S. Skrunes, C. E. Jones, C. Brekke, B. Holt, and A. P. Doulgeris, "Analysis of Evolving Oil Spills in Full-Polarimetric and HybridPolarity SAR,” IEEE Trans. Geosci. Remote Sens., vol. 55, no. 7, pp. 41904210, July 2017.

[12] S. Angelliaume, P. C. Dubois-Fernandez, C. E. Jones, B. Holt, B. Minchew, E. Amri and V. Miegebielle, "SAR Imagery for Detecting Sea 
Surface Slicks: Performance Assessment of Polarization-Dependent Parameters,” IEEE Trans. Geosci. Remote Sens., vol. PP, 2018.

[13] F. L. Penaranda-Foix, J. M. Catala-Civera, A. J. Canos-Marin and B. Garcia-Banos, "Circuital analysis of a coaxial re-entrant cavity for performing dielectric measurement”, in Proc. of the IMS2009, Boston, MA (USA) 2009, vol. 1 pp. 1309-1312.

[144] F. L. Penaranda-Foix, M. D. Janezic, J. M. Catala-Civera and A. J. Canos, "Full-Wave Analysis of Dielectric-Loaded Cylindrical Waveguides and Cavities Using a New Four-Port Ring Network,” IEEE Trans. Microw. Theory Techn., vol. 60, no. 9, Sep. 2012, pp. 2730-2740.

[15] F. L. Penaranda-Foix and Jose M. Catala-Civera, "Circuital analysis of cylindrical structures applied to the electromagnetic resolution of resonant cavities”, Chapter 7 in Passive Microwave Components and Antennas, 1st ed, Ed. IN-TECH, April 2010 (ISBN 978-953-307-083-4). Hardcopy and Online paper are available at webpage: http://sciyo.com/books/show/title/passivemicrowave-components-and-antennas.

[16] F. L. Penaranda-Foix, P. J. Plaza-Gonzalez, B. Garcia-Banos, and D. Polo-Nieves, "A nondestructive method of measuring the dielectric and magnetic properties of laminate materials in open cavities," in IEEE MTT-S Int. Microw. Symp. Dig., vol. 3. Jun. 2004, pp. 1821-1823.

[17] D. Marqués-Villarroya, F. L. Peñaranda-Foix, B. García-Baños, J. M. Catalá-Civera and J. D. Gutiérrez-Cano, "Enhanced Full-Wave Circuit Analysis for Modeling of a Split Cylinder Resonator". IEEE Trans. Microw. Theory Techn., vol. 65, no. 4, pp. 1191-1202, April 2017.

[18] L. Li and C. H. Liang, Analysis of resonance and quality factor of antenna and scattering systems using complex frequency method combined with model-based parameter estimation. Progress in Electromagnetic Research, PIER 46, 165-188, 2004.

[19] R.F. Harrington, Time Harmonic Electromagnetic Fields, New York, NY, USA: John Wiley \& Sons, 2001.

[20] A. J. Canós, J. M. Catalá-Civera, F. L. Peñaranda-Foix and E. de los Reyes-Davó, "A Novel Technicque for Deembedding the Unloaded Resonance Frequency From Measurements of Microwave Cavities,” IEEE Trans. Microw. Theory Techn., vol. 54, no. 8, Aug. 2006, pp. 3407-3416.

[21] http://www.qwed.com.pl/quickwave.html

[22] B. Durand, Kerogen, Insoluble Organic matter from Sedimentary Rocks, 1st ed., Technip Editions, Paris, France, 1980. ISBN: 2-7108-0371-2

[23] J. Doventon, Geological Log Analysis - Organic Mudstones, Kansas Geological Survey. [Online]

http://www.kgs.ku.edu/Publications/Bulletins/LA/10_mudstones.html [24] B. Ryan and D. Wells, Possible use of water isotherms to measure porosity and related properties of shales, Report for Canada Ministry of Energy, Mines and Petroleum Resources, 2009.

[25] G. R. Chalmers and M. R. Bustin, “ The Effects and Distribution of Moisture in Gas Shale Reservoir Systems," in Proc. AAPG Annual Convention and Exhibition, April, 2010, New Orleans, USA.

[26] K. B. Krauskopf, Introduction to geochemistry, 2nd ed., New York: McGraw-Hill, New York, 1979. ISBN: 0-07-035447-2.

[27] Evaluation of Measurement Data- Guide to the Expression of Uncertainty in Measurement, document JCGM Guide 100:2008, 2008. [Online]

https://www.bipm.org/utils/common/documents/jcgm/JCGM 100_2008_E.pd $\underline{\mathrm{f}}$

[28] M. Hiebel, Fundamentals of Vector Network Analysis, 5th ed., Rohde \& Schwarz GmbH \& Co, Munich, Germany, 2011. ISBN: 978-3-939837-06-0

[29] James Baker-Jarvis, Michael D. Janezic, Paul D. Domich, and Richard G. Geyer, "Analysis of an Open-Ended Coaxial Probe with Lift-off for Nondestructive Testing". IEEE Trans. On Instrumentation And Measurement, Vol. 43, NO. 5, October 1994, pp.711-718

[30] James Baker-Jarvis and Bill F. Riddle, "Dielectric Measurements Using a Reentrant Cavity: Mode-Matching Analysis". NIST Technical Note 1384, on line available at: https://www.gpo.gov/fdsys/pkg/GOVPUB-C134646cdf83eb3d85078c192b8fbc3fba9/pdf/GOVPUB-C134646cdf83eb3d85078c192b8fbc3fba9.pdf
[31] J. O. Alvarez, and F. Peñaranda-Foix, "Multi-frequency Microwave Resonance Cavity for Nondestructive Core Plug Measurements," in Proc. International Geoscience and Remote Sensing Symposium (IGARSS), July, 2018, Valencia, Spain.

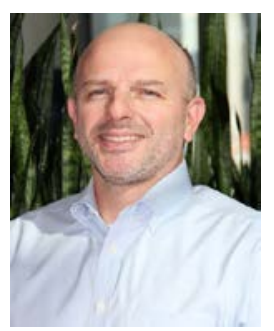

Jose Oliverio Alvarez (M'14-SM'19) received the B.S. (licenciatura) degree in mathematics from the Universidad del Valle de Guatemala, Guatemala City, Guatemala, in 1997, a Mathematics Diploma from ICTP, Trieste, Italy in 1998 and a Ph.D. degree in applied mathematics from the University of Arizona, Tucson, AZ, USA, in 2005. He also received a graduate certificate in digital signal processing from Purdue University, West Lafayette, IN, USA in 2013.

He was with ExxonMobil Upstream Research Company from 2006 to 2014. He is currently a Petroleum Engineer Specialist in the sensors development team at Aramco Research Center Houston. His research interests include microwave measurements, microwave characterization of geomaterials, inductive sensing, microwave and acoustic logging and borehole radar. Dr. Alvarez is a member of IEEE, the society of petrophysicists and well log analysts (SPWLA) and is a reviewer for international journals.

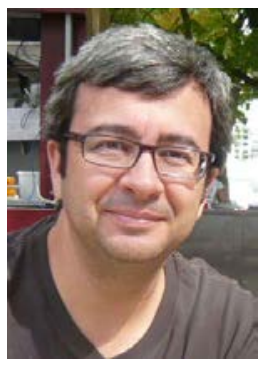

Felipe L. Peñaranda-Foix (M'92-SM'00) was born in Benicarló (Spain) in 1967. He received the M.S. degree in electrical engineering from Universidad Politécnica de Madrid, Madrid, Spain, in 1992, and the $\mathrm{Ph} . \mathrm{D}$. degree in electrical engineering from Universidad Politécnica de Valencia (UPV), Valencia, Spain, in 2001. In 1992, he joined the Departamento de Comunicaciones at UPV, where he is currently Associate Professor and also researcher at ITACA Institute at UPV. His current research interests include electromagnetic scattering, microwave circuits and cavities, sensors and microwave heating applications. He has co-authored more than 100 papers in referred journals and conference proceedings and more than 50 engineering reports for companies.

Dr. Peñaranda-Foix is IEEE and AMPERE member and reviewer of several international Journals.

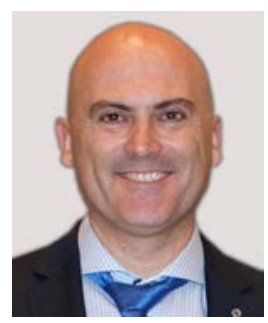

Jose M. Catalá-Civera was born in Valencia (Spain) in February 1969. He received the Dipl. Ing. and Ph.D. degrees from the Universidad Politécnica de Valencia, Spain, in 1993 and 2000, respectively. Since 1996, he has been with the Communications Department, Universidad Politécnica de Valencia, where he received the Readership in 2000 becoming full professor in 2011. Currently he is head of the Microwave Applications Research Division of the Institute ITACA at the 
Universidad Politécnica de Valencia. His research interests encompass the design and application of microwave theory and applications, the use of microwaves for electromagnetic heating, microwave cavities and resonators, measurement of dielectric and magnetic properties of materials and development of microwave sensors for non-destructive testing. He has co-authored about 100 papers in referred journals and conference proceedings, more than 50 engineering reports for companies and he holds 13 patents. Dr. Catala-Civera is IEEE Member, IMPI Member, he is reviewer of several international Journals and is currently Board Member of the Association of Microwave Power in Europe for Research and Education (AMPERE), a European-based organization devoted to the promotion of RF and microwave energy.

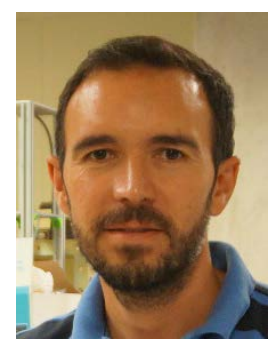

José D. Gutiérrez was born in Molinicos (Albacete), Spain, in 1984. He received the Dipl.Eng. in electrical engineering from the Universidad Politécnica de Valencia (UPV), Valencia, Spain, in 2008. In 2008, he joined the microwave division in the Institute for the Information and Communication Technologies (ITACA), UPV, as a Research and Development Engineer and as a Laboratory Technician. His current research interests include microwave measurement techniques, devices for the electromagnetic characterization of materials and industrial microwave processes. 